CERN-TH.7494/94

Imperial/TP/94-95/3

hep-th/9411099

revised

\title{
Constant magnetic field in closed string theory: an exactly solvable model
}

\author{
J.G. Russo* \\ Theory Division, CERN \\ CH-1211 Geneva 23, Switzerland \\ and \\ A.A. Tseytlin ${ }^{\star \dagger}$ \\ Theoretical Physics Group, Blackett Laboratory \\ Imperial College, London SW7 2BZ, U.K.
}

\begin{abstract}
We consider a simple model describing a closed bosonic string in a constant magnetic field. Exact conformal invariance demands also the presence of a non-trivial metric and antisymmetric tensor (induced by the magnetic field). The model is invariant under target space duality in a compact Kaluza-Klein direction introduced to couple the magnetic field. Like open string theory in a constant gauge field, or closed string theory on a torus, this model can be straightforwardly quantized and solved with its spectrum of states and partition function explicitly computed. Above some critical value of the magnetic field an infinite number of states become tachyonic, suggesting a presence of phase transition. We also construct heterotic string generalisations of this bosonic model in which the constant magnetic field is embedded either in the Kaluza-Klein or internal gauge group sector.
\end{abstract}

November 1994

\footnotetext{
* e-mail address: jrusso@vxcern.cern.ch

* e-mail address: tseytlin@ic.ac.uk

$\dagger$ On leave from Lebedev Physics Institute, Moscow, Russia.
} 


\section{Introduction}

The study of simple exactly solvable models may provide a way to understand vacuum properties in string theory and discover its hidden symmetries. In the case of open string theory, the simplest example of a non-trivial background is a constant abelian gauge field. It represents a conformally invariant theory, and various aspects of string theory can be consistently analysed with a constant field strength as a free external parameter. For example, one can easily find the exact tree-level (disc) expression for the partition function $Z_{0}$ and (since in the open string theory the massless effective action $S$ is proportional to the renormalised value of $Z_{0}$ [1].2]) discover that the $\partial F$ - independent part of the exact tree-level $S(F)$ is given by the Born-Infeld action. One can also compute find the 1-loop (annulus) correction to the partition function [1, 3] and study the open string behaviour in the constant electric or magnetic fields (e.g. analyse tachyonic instabilities and existence of a critical field [4], string pair creation by an electric field [5], phase transition in a magnetic field [6], dependence of the Hagedorn temperature on the electric field [7], etc.).

In this open string model the target space metric is the Minkowski one, i.e. the deformation of the geometry caused by the presence of the electromagnetic stress-energy is ignored. This is a good approximation for weak fields much smaller than the Planck scale ones. It would be very interesting to include the back reaction, i.e. to treat the geometry and the gauge field on the same footing. Unfortunately, in the case of the open string theory a complete treatment requires including higher loop orders (to incorporate the bound-state closed string sector) and is hardly tractable.

Given that the physically relevant string models are expected to be closed string ones [8,9] a natural question is whether there exists a simple solvable analogue of the constant abelian gauge field problem in the closed bosonic, superstring and heterotic string theories. It is known how to incorporate some simple types of external parameters (e.g. constant moduli of a target space torus or a temperature) in closed string theory. Here we suggest how to include another one, representing the constant magnetic field strength.

One may wonder how important is actually the influence of the magnetic field on the geometry. In principle, the new scale $f^{-1}$ introduced by the magnetic field $\left(\mathcal{H}=R^{-1} f\right)$ and can be arbitrary. In practice, to study non-trivial stringy effects one is to consider $f^{-1}$ to be of a Planck scale. In fact, the present model is a "conformal improvement" of the one investigated in [10,11] where the change in the geometry due to the presence of the magnetic field was ignored. In ref. [11] it was observed that the corresponding thermodynamic partition function of a string gas in a magnetic field diverges if $f$ exceeds some critical value. It was suggested that a phenomenon analogous to the Meissner effect in type-I superconductors (leading to a critical curve on the magnetic field - temperature diagram), where strong fields restore the hidden symmetry of the theory, may take place in string theory. This was supported by the appearance of infinitely many extra massless states at a critical value of the field (the emergence of tachyons at strong magnetic fields 
was found also in the open string model [6]). The critical magnetic field has Planck value $\left(f \sim 1 / \sqrt{\alpha^{\prime}}\right)$, i.e. it produces a a strong deformation of the geometry, with the curvature being proportional to $f^{2}$. That means the back reaction cannot be ignored, and to address these important issues one is to repeat the analysis starting with a consistent conformally invariant model.

To couple an external magnetic field (described by a vector potential $A_{i}$ ) to a closed bosonic model one may follow the Kaluza-Klein idea, introducing a periodic "internal" coordinate $\phi$ (compactified on a circle of radius $R$ ) and adding the terms $(\partial \phi)^{2}$ and $A_{i} \partial x^{i} \partial \phi$ to the free string Lagrangian (in the heterotic string case the role of $\phi$ may be played by a Weyl fermion of an internal gauge sector). The resulting $\sigma$-model is not, however, conformally invariant. This is not surprising since the energy of the magnetic field should modify the space-time geometry in the non-compact $\left(t, x^{i}\right)$ directions. To find a conformally invariant model, one should start with the full string (Einstein-Maxwell-type) equations, plug in the ansatz for the magnetic field, determine the corresponding metric, antisymmetric tensor and dilaton, then go to the next order in $\alpha^{\prime}$, etc.

Remarkably, there exists a simple conformal model [12] which gives an exact solution to this seemingly complicated back reaction problem. It has a curved metric parametrised by $A_{i}=-\frac{1}{2} F_{i j} x^{j}$ (which is nothing but the metric of the product of the group space of the Heisenberg group and a line) and the antisymmetric tensor proportional to $F_{i j}$ (and constant dilaton). Introducing the "light-cone" coordinates $v=\phi+t, u=\phi-t$ one learns that the corresponding string Lagrangian is formally ( $\phi$ is periodic!) of a "plane- fronted wave" type, i.e. has a null covariantly constant Killing vector (see e.g. [13, 14, 15, 16]) and, provided the Maxwell equation $\partial_{i} F^{i j}=0$ is satisfied, represents a string theory solution to all orders in $\alpha^{\prime}$ [12]. In the limit of non-compact $\phi(R \rightarrow \infty)$ in addition to having "plane wave" interpretation, this model is equivalent [12] to a WZW model based on a non-semisimple group (with the basic $D=4$ example being the model of ref. [17], see also $[18,19,20,21,22,23]$ ).

The purpose of the present paper is to investigate the properties of this model by solving it explicitly, i.e. computing the spectrum of states and the partition function. This model is easier to solve than, e.g., WZW models based on simple groups; in particular, the possibility of fixing the light-cone gauge demonstrates its unitarity. We shall confirm the presence of the tachyon instabilities and a phase transition for a critical value of the magnetic field. We expect that it should be possible to determine the scattering amplitudes and thus an effective action for extra "massless" fields that emerge at the critical magnetic field. That may help to unravel new string symmetries.

The essential part of the definition of our model is that $\phi$ is a "Kaluza-Klein" coordinate, i.e. is compactified on a circle of radius $R$. Interestingly, the target space duality symmetry [24] interchanging momentum and winding modes and $R$ with $\alpha^{\prime} / R$ which is present in the free theory remains an exact symmetry of this model (in particular, of 
its spectrum and the partition function) for arbitrary magnetic field. Another important property is that while in Kaluza-Klein theory the role of charge is played by the linear momentum in a compact direction, here the "charge" (to which the magnetic field is coupled in the mass formula) is represented by the sum of the "left" string momentum $Q$ (equal to the duality invariant combination of the linear and winding momenta) and the energy $E$ of the string. The appearance in the string mass formula of the term $E f$ (in addition to $Q f)$ which is linear in the energy is a direct consequence of the non-flat geometry of our background (or, equivalently, of its conformal invariance).

The plan of this paper is the following. In Section 2 we describe some general aspects of the model, including its geometrical interpretation, relation to non-semisimple WZW models, target space duality, point-particle limit and explicit solution of the tachyon equation.

In Section 3 we solve the classical string equations, express the solution in terms of the free fields, perform the complete quantisation of the theory in the "light-cone" gauge and analyse the resulting spectrum of states.

Section 4 describes the calculation of the partition function on the torus, first starting from the path integral and then using the operator approach (with equivalent results). We discuss the new singularities in the partition function related to the presence of tachyon instabilities in the spectrum.

In Section 5 we discuss the supersymmetric extensions of the bosonic model. In particular, we construct the exact conformal heterotic string models in which the constant magnetic field is embedded either in the bosonic (Kaluza-Klein) or in the fermionic (internal gauge group) sector. Some conclusions and open problems are presented in Section 6 .

\section{Description of the model}

\subsection{Abelian vector coupling in closed string theory}

To motivate the discussion of our specific string model let us consider the following problem: how to "embed" a spatial (or magnetic) part of Maxwell equations in closed string theory? A simple application of the Kaluza-Klein idea leads to the following model (see e.g. [10])

$$
\begin{gathered}
I=\frac{1}{4 \pi \alpha^{\prime}} \int d^{2} \sigma\left[\sqrt { g } \left(-\partial_{\alpha} t \partial^{\alpha} t+\partial_{\alpha} x^{i} \partial^{\alpha} x_{i}+\partial_{\alpha} \phi \partial^{\alpha} \phi\right.\right. \\
\left.\left.\quad+2 A_{i}(x) \partial_{\alpha} x^{i} \partial^{\alpha} \phi\right)+2 i A_{i}(x) \epsilon^{\alpha \beta} \partial_{\alpha} x^{i} \partial_{\beta} \phi\right]
\end{gathered}
$$

where $x^{\mu}=\left(t, x^{i}\right)$ are coordinates of a flat space-time $(i=1, \ldots, D-2)$ and $\phi$ is a compact "internal" coordinate used to couple the gauge field to the string. The vector and axial gauge fields corresponding to the "off-diagonal" $\left(x^{\mu} \phi\right)$-components of the metric and the 
antisymmetric tensor field are taken to be equal. As a result, only the "right" part of $x^{i}$ is coupled to $A_{i}$, i.e. has a gauge charge. I In the conformal gauge, the Lagrangian density corresponding to action $(2.1)$ becomes $\left(I \equiv \frac{1}{\pi \alpha^{\prime}} \int d^{2} \sigma L\right)^{2}$

$$
L=-\partial t \bar{\partial} t+\partial \phi \bar{\partial} \phi+2 A_{i}(x) \bar{\partial} x^{i} \partial \phi+\partial x^{i} \bar{\partial} x^{i}
$$

Eq. (2.2) does not, however, represent a conformally invariant model, i.e. such vector coupling does not satisfy the string equations, implying that one needs to add also other background fields.

It is remarkable that a conformal model can be obtained by a simple modification of (2.2), introducing non-trivial metric and the antisymmetric tensor components in the " $i 0$ " direction,

$$
\begin{aligned}
& I=\frac{1}{4 \pi \alpha^{\prime}} \int d^{2} \sigma\left[\sqrt { g } \left(-\partial_{\alpha} t \partial^{\alpha} t+\partial_{\alpha} x^{i} \partial^{\alpha} x_{i}+\partial_{\alpha} \phi \partial^{\alpha} \phi\right.\right. \\
& \left.\left.+2 A_{i}(x) \partial_{\alpha} x^{i} \partial_{\beta}(\phi-t)\right)+2 i A_{i}(x) \epsilon^{\alpha \beta} \partial_{\alpha} x^{i} \partial_{\beta}(\phi-t)\right]
\end{aligned}
$$

As a result, the $x^{\mu}$-part of the model is no longer flat, with non-trivial metric and antisymmetric tensor "compensating" for the distortion of the geometry by the magnetic field. The conformal gauge Lagrangian corresponding to eq. (2.3) takes the form

$$
L=-\partial t \bar{\partial} t+\partial \phi \bar{\partial} \phi+2 A_{i}(x) \bar{\partial} x^{i}(\partial \phi-\partial t)+\partial x^{i} \bar{\partial} x^{i}
$$

i.e. (up to a total derivative)

$$
\begin{gathered}
L=\partial u \bar{\partial} v+2 A_{i}(x) \bar{\partial} x^{i} \partial u+\partial x^{i} \bar{\partial} x^{i} \\
u \equiv \phi-t, \quad v \equiv \phi+t
\end{gathered}
$$

The action is invariant under a gauge transformation of $A_{i}$ combined with a coordinate transformation of $v$

$$
A_{i}^{\prime}=A_{i}+\partial_{i} \alpha, \quad v^{\prime}=v-2 \alpha, \quad \alpha=\alpha(x)
$$

1 In this sense such model is a bosonic analogue of the heterotic string. Assuming that $\phi$ can be fermionised (and adding extra free degrees of freedom) one may also directly embed this model into the heterotic string theory (see Section 5).

2 Here we consider the Euclidean world sheet and use the following notation: $\partial=\frac{1}{2}\left(\partial_{1}-\right.$ $\left.i \partial_{2}\right), \bar{\partial}=\frac{1}{2}\left(\partial_{1}+i \partial_{2}\right), z=\sigma_{1}+i \sigma_{2}, \bar{z}=\sigma_{1}-i \sigma_{2}, d^{2} \sigma \equiv d \sigma_{1} d \sigma_{2}$. In the case of the Minkowski world sheet used in Section $3 \partial$ and $\bar{\partial}$ should be replaced by $\partial_{+}=\frac{1}{2}\left(\partial_{\tau}+\partial_{\sigma}\right)$, and $\partial_{-}=\frac{1}{2}\left(\partial_{\tau}-\partial_{\sigma}\right)$, and $z, \bar{z}$ by $\sigma_{ \pm}=\tau \pm \sigma$, and the overall sign of the action should be reversed. 
This model belongs to the general class of models considered in [12] (see also [14]) and represents an exact conformal field theory (to all orders in $\alpha^{\prime}$ ) provided $A_{i}$ satisfies the magnetic part of the Maxwell's equations in flat space $3^{3}$

$$
\partial_{i} F^{i j}=0, \quad F_{i j} \equiv \partial_{i} A_{j}-\partial_{j} A_{i}
$$

In the main part of this paper we will study a particular model with $A_{i}=-\frac{1}{2} f \epsilon_{i j} x^{j},(i, j=$ $1,2)$, representing a constant magnetic field in 3-space.

Since $A_{i}$ is dimensionless like a component of the metric, $f$ has an inverse length dimension. The "physical" (dimension $\mathrm{cm}^{-1}$ ) vector field is obtained by dividing $A_{i}$ over the radius $R$ of the compact dimension $\phi$, i.e. the proper magnetic field is $\mathcal{H}=f R^{-1}$ (for simplicity in what follows we shall still call $f$ the magnetic field). $f^{-1}$ defines another scale of our model which is independent of $R$ and $\alpha^{\prime}$ (in the case of a heterotic model with the magnetic field in the internal gauge sector the scale $R$ will be absent, or, effectively, $R=$ $\sqrt{\alpha^{\prime} / 2}$, see Section 5). Since the curvature and the antisymmetric tensor field strengths corresponding to our model satisfy $R \sim H^{2} \sim F^{2} \sim f^{2}$, by a choice of $f$ their scale can be made arbitrarily larger than the Planck scale $\left(\sqrt{\alpha^{\prime}}\right)$.

The $D$-dimensional background corresponding to (2.5) is a mixture of a curved metric $G_{M N}$ and the antisymmetric tensor $B_{M N}\left(x^{M}=\left(t, x^{i}, \phi\right)\right)$. The metric

$$
d s^{2}=G_{M N} d x^{M} d x^{N}=d u d v+2 A_{i}(x) d x^{i} d u+d x^{i} d x_{i}
$$

${ }^{3}$ One may also consider the model [12] $L=\partial u^{\prime} \bar{\partial} v^{\prime}+k \partial u^{\prime} \bar{\partial} u^{\prime}+2 A_{i}(x) \bar{\partial} x^{i} \partial u^{\prime}+\partial x^{i} \bar{\partial} x_{i}, \quad k=$ const . The two models are formally related $\left(v=v^{\prime}+k u^{\prime}, u=u^{\prime}\right)$ as in the case of non-compact coordinates (the dependence on $k$ can be absorbed into a rescaling of $\alpha^{\prime}$ in the physical quantities like partition function).

4 A simple reason why the corresponding $\sigma$-model (2.5) is conformal is the following (for a rigorous argument see [12]). If one first does not introduce a source coupled to $v$, the path integral over $v$ imposes a constraint on $u$ and thus gives a free theory for $x^{i}$. It remains then to check conformal invariance in the $u u$ and $u i$ directions. The corresponding $\beta$-functions can not contain $\alpha^{\prime}$-corrections since $L / \alpha^{\prime}$ (with $A_{i}$ quadratic in $x^{i}$ ) is invariant under the simultaneous rescaling $v \rightarrow \lambda^{2} v, x^{i} \rightarrow \lambda x^{i}$ and $\alpha^{\prime} \rightarrow \lambda^{2} \alpha^{\prime}$. Thus $\alpha^{\prime}$ can be given an arbitrary value by a coordinate transformation but the $\beta$-functions are assumed to be covariant tensors transforming under the above rescalings in the standard way (notice that $u$ is not rescaled). To see why the 1-loop conformal invariance conditions are satisfied one may use the observation that this model is formally (ignoring the issue of periodicities of coordinates) dual to a flat space model, see Section 2.4 . 
is of a plane-fronted wave type, i.e. has one covariantly constant Killing vector.5 The generalised connection which enters the string propagation equation

$$
\begin{gathered}
\partial \bar{\partial} x^{L}+\hat{\Gamma}_{-M N}^{L}(x) \partial x^{M} \bar{\partial} x^{N}=0 \\
\hat{\Gamma}_{ \pm M N}^{L}=\Gamma_{M N}^{L} \pm \frac{1}{2} H_{M N}^{L}, H_{L M N}=3 \partial_{[L} B_{M N]}=\left\{H_{u i j}=F_{i j}, 0\right\}
\end{gathered}
$$

has the following non-zero components: $\hat{\Gamma}_{-u j}^{i}=-F^{i}{ }_{j}, \hat{\Gamma}_{-i j}^{v}=2 \partial_{i} A_{j}, \quad \hat{\Gamma}_{-u i}^{v}=-2 A^{j} F_{i j}$. The only non-trivial components of the corresponding generalised curvature tensor and Ricci tensor are

$$
\hat{R}_{-i j k}^{v}=-2 \hat{R}_{-u j k}^{i}=2 \partial_{i} F_{j k}, \quad \hat{R}_{-u i}=-\partial_{j} F^{j}{ }_{i}
$$

If $A_{i}$ satisfies (2.7) the space is thus Ricci-flat in generalised sense $\left(\hat{R}_{M N}=0\right.$, i.e. $R_{M N}=$ $\frac{1}{4} H_{M K L} H_{N}^{K L}, \nabla_{K} H^{M N K}=0$ ). Moreover, if $A_{i}$ has a constant field strength, it is parallelisable, i.e. $\hat{R}_{N K L}^{M}=0.0$ The resulting space is, in fact, a group space of a non-semisimple group, see [12] and below. Introducing a vierbein and computing the components of the Lorentz connection one finds that for $F_{i j}=$ const., $d e^{m}+\omega_{n}^{m} e^{n}=0$ can be identified with the Maurer-Cartan equation from which one can read off the structure constants (proportional to $F_{i j}$ ) of the corresponding non-semisimple algebra.

\subsection{Constant field strength case and relation to non-semisimple $W Z W$ models}

In what follows we shall specialise to the case of a constant magnetic field strength

$$
A_{i}=-\frac{1}{2} F_{i j} x^{j}, \quad F_{i j}=\text { const }, \quad i, j=1, \ldots, D-2
$$

When $u, v$ are non-compact, the spaces corresponding to (2.5),(2.12) can be interpreted 12] as "boosted" products of group spaces, or, equivalently, as group spaces corresponding to non-semisimple groups [17] (see also [19,20]). This can be demonstrated by first putting $F_{i j}$ (by a rotation of $x^{i}$ ) into a block-diagonal form, so that its elements are represented by constants $f_{1}, \ldots, f_{[D / 2-1]}$. The Lagrangian is then (we split $x^{i}$ into pairs representing 2-planes)

$$
L=\partial u \bar{\partial} v+F_{i j} x^{i} \bar{\partial} x^{j} \partial u+\partial x^{i} \bar{\partial} x^{i}
$$

5 The inverse metric is $G^{u v}=2, G^{u i}=G^{u u}=0, G^{v i}=-2 A^{i}, G^{v v}=4 A_{i} A^{i}, G^{i j}=$ $\delta^{i j}, \operatorname{det} G^{-1}=-4$.

6 The standard curvature of the metric contains extra $\partial F$ and $F^{2}$-terms.

7 It should be noted that the parallelisability is a natural string theory generalisation of the flatness property in the point-particle theory case. 


$$
=\partial u \bar{\partial} v+\sum_{s=1}^{[D / 2-1]}\left(f_{s} \epsilon_{i_{s} j_{s}} x^{i_{s}} \partial u \bar{\partial} x^{j_{s}}+\partial x^{i_{s}} \bar{\partial} x_{i_{s}}\right) .
$$

The first non-trivial case is that of $D=4$, i.e. $F_{i j}=f \epsilon_{i j}$. The corresponding model $\left(x_{1}=r \cos \theta, x_{2}=r \sin \theta\right)$

$$
\begin{gathered}
L=\partial u \bar{\partial} v+f \epsilon_{i j} x^{i} \bar{\partial} x^{j} \partial u+\partial x^{i} \bar{\partial} x_{i} \\
=\partial u \bar{\partial} v+f r^{2} \bar{\partial} \theta \partial u+\partial r \bar{\partial} r+r^{2} \partial \theta \bar{\partial} \theta,
\end{gathered}
$$

is equivalent to the $E_{2}^{c}$ WZW model of ref. [17]. In fact, if $\phi=\frac{1}{2}(u+v)$ is assumed to be non-compact, then $f$ can be set equal, e.g., to -1 by a rescaling of $u, v$. The coordinate transformation

$$
x_{1}=y_{1}+y_{2} \cos u, \quad x_{2}=y_{2} \sin u, \quad v=v^{\prime}+y_{1} y_{2} \sin u
$$

puts (2.14) in the form (up to a total derivative term) 8

$$
\begin{gathered}
L=\partial u \bar{\partial} v^{\prime}+\partial y_{1} \bar{\partial} y_{1}+\partial y_{2} \bar{\partial} y_{2}+2 \cos u \partial y_{2} \bar{\partial} y_{1} \\
\equiv \partial u \bar{\partial} v^{\prime}+\left(g_{i j}+b_{i j}\right)(u) \partial y^{i} \bar{\partial} y^{j}
\end{gathered}
$$

which is obtained from the $R \times S U(2)$ WZW action by a singular boost and rescaling of the level $k$ or $\alpha^{\prime}$ (see 20,22]).

All higher $D$ models are related [12] to similar WZW models based on direct products of $S L(2, R)_{-k}, S U(2)_{k}$ and $R$ factors, or, equivalently, on corresponding non-semisimple groups. The parameters $f_{s}$ are essentially the rescaled levels $k_{n}$ of the factors.

In the rest of this paper we shall consider the simplest $D=4$ model (2.14); however, all our results (exact solution of the string equations, light-cone quantisation, spectrum, partition function) can be straightforwardly generalised to the case of (2.13) with an arbitrary D.

In order to describe the interaction of a closed string with a magnetic field we have assumed that $\phi$ is compactified on a circle with period $2 \pi R$. In the limit $R \rightarrow \infty$ our model is equivalent to the WZW model of [17]. Though the construction of [17] of a nonsemisimple WZW model formally goes through also in the case of a periodic $\phi=\frac{1}{2}(u+v)$ we shall not use the current algebra relations in the explicit solution of our model in Sections 3 and 4.9

8 The Lagrangian (2.14) can be put also in the following equivalent form (see e.g. [21]) $L=$ $\partial u \bar{\partial} v+f x^{2} \partial u \bar{\partial} u+f \epsilon_{i j} x^{i}\left(\partial u \bar{\partial} x^{j}-\partial x^{j} \bar{\partial} u\right)+\partial x_{i} \bar{\partial} x^{i}$.

9 The current algebra approach to the solution of the model of [17] in the case of a non-compact $\phi$ was developed in [18,25]. 


\subsection{Dimensional reduction interpretation}

In trying to couple an abelian gauge field to a closed string theory in a conformally invariant way we have suggested the simple model (2.5). In order to further clarify the "magnetic field" interpretation of $A_{i}$ let us reinterpret this model in the Kaluza-Klein fashion, assuming that $\phi$ is a compact internal dimension (see also the discussion in [12]).

One can rewrite the Lagrangian (2.5) in the form

$$
\begin{gathered}
L=-\left[\partial t+A_{i}(x) \partial x^{i}\right]\left[\bar{\partial} t+A_{i}(x) \bar{\partial} x^{i}\right]+\partial x^{i} \bar{\partial} x_{i}+A_{i}(x)\left(\partial x^{i} \bar{\partial} t-\bar{\partial} x^{i} \partial t\right) \\
+\left[\partial \phi+A_{i}(x) \partial x^{i}\right]\left[\bar{\partial} \phi+A_{i}(x) \bar{\partial} x^{i}\right]-A_{i}(x)\left(\partial x^{i} \bar{\partial} \phi-\bar{\partial} x^{i} \partial \phi\right)
\end{gathered}
$$

For definiteness, we shall assume that we start with the $D=5$ model and represent it as $D=4$ model with extra vector couplings. Interpreting $\phi$ as a "fifth" internal coordinate we find the corresponding four dimensional background

$$
\begin{gathered}
d s_{4}^{2}=-\left[d t+A_{i}(x) d x^{i}\right]^{2}+d x_{i} d x^{i}, \quad B_{i t}=A_{i}(x) \\
\mathcal{A}_{i}=-\mathcal{B}_{i}=A_{i}(x)
\end{gathered}
$$

In addition to a curved metric and antisymmetric tensor backgrounds there are also vector $\mathcal{A}_{i}$ and axial-vector $\mathcal{B}_{i}$ fields equal (up to sign) to $A_{i}$. When $A_{i}$ satisfies (2.7) this is an exact (all-order in $\alpha^{\prime}$ ) solution corresponding to the $D=5$ bosonic string effective action for $G_{M N}, B_{M N}$ and dilaton $\Phi$ dimensionally reduced to $D=4$

$$
\begin{gathered}
S_{4}=\int d^{4} x \sqrt{\hat{G}} e^{-2 \Phi+\sigma}\left[\hat{R}+4\left(\partial_{\mu} \Phi\right)^{2}-4 \partial_{\mu} \Phi \partial^{\mu} \sigma\right. \\
\left.-\frac{1}{12}\left(\hat{H}_{\mu \nu \lambda}\right)^{2}-\frac{1}{4} e^{2 \sigma}\left(\mathcal{F}_{\mu \nu}(\mathcal{A})\right)^{2}-\frac{1}{4} e^{-2 \sigma}\left(\mathcal{F}_{\mu \nu}(\mathcal{B})\right)^{2}+O\left(\alpha^{\prime}\right)\right],
\end{gathered}
$$

where we have defined $G_{55} \equiv e^{2 \sigma}$ and

$$
\begin{gathered}
\mathcal{F}_{\mu \nu}(\mathcal{A})=2 \partial_{[\mu} \mathcal{A}_{\nu]}, \quad \mathcal{F}_{\mu \nu}(\mathcal{B})=2 \partial_{[\mu} \mathcal{B}_{\nu]}, \quad \mathcal{A}_{\mu} \equiv G^{55} G_{\mu 5}, \quad \mathcal{B}_{\mu} \equiv B_{\mu 5} \\
\hat{G}_{\mu \nu} \equiv G_{\mu \nu}-G_{55} \mathcal{A}_{\mu} \mathcal{A}_{\nu}, \quad \hat{H}_{\lambda \mu \nu}=3 \partial_{[\lambda} B_{\mu \nu]}-3 \mathcal{A}_{[\lambda} \mathcal{F}_{\mu \nu]}(\mathcal{B})
\end{gathered}
$$

Since $\mathcal{A}_{i}$ and $-\mathcal{B}_{i}$ are equal on our solution (this is directly related to the fact that the scalars $\Phi$ and $\sigma$ are constant), they can be treated as a single vector field. In fact, assuming that $\Phi=$ const, $\sigma=0$ and introducing the two vectors $V_{\mu}=\frac{1}{2}\left(\mathcal{A}_{\mu}-\mathcal{B}_{\mu}\right)$ and $W_{\mu}=$ $\frac{1}{2}\left(\mathcal{A}_{\mu}+\mathcal{B}_{i}\right)$ we get the effective action

$$
S_{4}=k_{0} \int d^{4} x \sqrt{\hat{G}}\left[\hat{R}-\frac{1}{12}\left(\hat{H}_{\mu \nu \lambda}\right)^{2}-\frac{1}{2}\left(\mathcal{F}_{\mu \nu}(V)\right)^{2}-\frac{1}{2}\left(\mathcal{F}_{\mu \nu}(W)\right)^{2}+O\left(\alpha^{\prime}\right)\right]
$$


We conclude that the solution (2.19) describes a curved space-time with the antisymmetric tensor $\hat{H}_{t i j}=H_{t i j}=F_{i j}$ and one non-vanishing vector field $V_{\mu}=\left(0, A_{i}\right)$ with the magnetic field strength $F_{i j}$.

Introducing the vierbein basis $e^{m}, m=(\hat{0}, \hat{i})$ for the metric (2.19)

$$
e^{\hat{0}}=d t+A_{i}(x) d x^{i}, \quad e^{\hat{i}}=d x^{i}
$$

one finds that the components of the Lorentz connection are

$$
\omega_{\hat{i}}^{\hat{0}}=\frac{1}{2} F_{i j} e^{\hat{j}}, \quad \omega_{\hat{i} \hat{j}}=\frac{1}{2} F_{i j} e^{\hat{0}}
$$

When $F_{i j}=$ const we thus get the Maurer-Cartan equation: $d e^{l}=-\frac{1}{2} C_{m n}^{l} e^{m} \wedge e^{n}$, $C_{\hat{i} \hat{j}}^{\hat{0}}=-F_{i j}, C_{m n}^{\hat{k}}=0$. The dual basis of the vector fields thus satisfies the Heisenberg algebra (it can be put into a canonical form by "diagonalising" $F_{i j}$ as in (2.13))

$$
\left[E_{i}, E_{j}\right]=-F_{i j} E_{0}, \quad\left[E_{i}, E_{0}\right]=0, \quad F_{i j}=\text { const }
$$

The metric (2.19) thus represents the group space of the Heisenberg group (or its product with $R$ in the case when $D-1$ is odd). 10 This of course is directly related to the fact that the higher dimensional metric (2.8) of which (2.19) is a dimensional reduction represents a group space of a non-semisimple group.

The magnetic field is constant in the preferred frame chosen in (2.19) in which the metric is stationary. As follows from (2.24) the field strength $\mathcal{F}_{\mu \nu}(V)=\left(0, F_{i j}\right)$ is actually covariantly constant $\left(F_{\hat{t} \hat{i}}=0, F_{\hat{i} \hat{j}}=F_{i j}, \omega_{\hat{i} \hat{k}} F_{\hat{k} \hat{j}}+\omega_{\hat{j} \hat{k}} F_{\hat{i} \hat{k}}=0\right)$. The metric (2.19) can be arbitrarily close to the flat one depending on the strength of the magnetic field. It is not asymptotically flat $\left(R \sim F^{2}\right)$ for non-zero $F_{i j}$ (i.e. it is supposed to describe only the region of space-time where the magnetic field is uniform).

One may wonder if there are similar uniform magnetic field solutions of (2.20) without antisymmetric tensor field. The answer is no if one does not introduce a non-trivial dilaton background. The reason is that in addition to the Eistein-type equation, one is to satisfy the dilaton equation that follows from (2.20). 11 This is impossible in terms of metric and vector field backgrounds only.

To give an explicit example of the solution (2.19) let us consider with the $D=5$ model $\left(x^{M}=\left(t, \phi, x^{i}, x^{3}\right), i=1,2\right)$ with $A_{i}=-\frac{1}{2} f \epsilon_{i j} x^{j}, A_{3}=0$ representing a generic constant

10 We are grateful to G. Gibbons for this remark.

11 There exists the axially symmetric static leading-order solution [26] which is the dilatonic generalisation of the Melvin solution. In this "flux-tube" type solution there is no antisymmetric tensor background but the magnetic field and dilaton decrease in the transverse directions [55]. 
magnetic field in 3-space. Then the $D=4$ metric for the uniform magnetic field solution $\left(V_{i}=A_{i}\right)$ is simply (cf. (2.14)

$$
d s_{4}^{2}=-\left(d t+\frac{1}{2} f r^{2} d \theta\right)^{2}+d r^{2}+r^{2} d \theta^{2}+d x_{3}^{2},
$$

i.e. is the direct product of a $D=3$ space-time (group space of the 3 -dimensional Heisenberg group) and a line and describes a "rotating universe" [12. 12 The non-trivial $D=3$ part of the metric $(\overline{2.26})$ satisfies

$$
R_{\mu \nu}=\frac{1}{4} H_{\mu \lambda \rho} H_{\nu}^{\lambda \rho}+\mathcal{F}_{\mu \lambda}(V) \mathcal{F}_{\nu}^{\lambda}(V) .
$$

In the absence of the gauge field contribution the only solution in $D=3$ is the anti de Sitter space (and its analytic continuation or topological modifications). In fact, in $D=3$ $H_{\mu \lambda \rho} \sim \epsilon_{\mu \lambda \rho}, H_{\mu \lambda \rho} H_{\nu}{ }^{\lambda \rho} \sim G_{\mu \nu}$. However, $\mathcal{F}_{\mu \lambda} \mathcal{F}_{\nu}{ }^{\lambda}$ and thus $R_{\mu \nu}$ are no longer proportional to $G_{\mu \nu}$. In the vierbein basis $e^{\hat{0}}=d t+\frac{1}{2} f r^{2} d \theta, e^{\hat{1}}=d r, e^{\hat{2}}=r d \theta, e^{\hat{3}}=d x^{3}$, one finds the following non-vanishing vierbein components of the Riemann and Ricci tensors corresponding to the homogeneous Heisenberg group space:

$$
R_{\hat{0} \hat{1} \hat{0} \hat{1}}=R_{\hat{0} \hat{2} \hat{0} \hat{2}}=\frac{1}{3} R_{\hat{1} \hat{2} \hat{1} \hat{2}}=\frac{1}{4} f^{2}, \quad R_{\hat{0} \hat{0}}=R_{\hat{1} \hat{1}}=R_{\hat{2} \hat{2}}=\frac{1}{2} f^{2}, \quad R_{\mu \nu} R^{\mu \nu}=\frac{3}{4} f^{4} .
$$

In Sections 3,4 we shall analyse this solution starting directly with the original $D=5$, or, equivalently (disregarding the trivial $x^{3}$-dimension) $D=4$ model (2.5) itself, and not with the "dimensionally reduced" background (2.19).

\subsection{Target space duality invariance}

The spectrum and the $S$-matrix of the bosonic string theory in flat space with one compact dimension $\phi$ are invariant under the duality symmetry which interchanges the winding and momentum modes corresponding to $\phi$ and, at the same time, inverts the radius, $R \rightarrow \alpha^{\prime} R^{-1}$. It is interesting that the inclusion of the gauge field coupling according to (2.3) or (2.5) preserves the duality invariance. We shall now show that the model (2.5) is "self-dual", i.e. is invariant under the $\sigma$-model duality [27] with $A_{i}$ not transforming (see also [12]). 3 We shall also see the duality symmetry explicitly in the spectrum of our model derived in Section 3, and in the partition function computed in Section 4.

12 A different reduction of the model of [17] giving $D=3$ plane-wave type background was considered in [22].

13 The $\sigma$-model duality invariance property is true also for the dimensionally reduced background (2.19) with respect to duality in $t$ direction. 
Starting with (2.4) and gauging the isometry in the $\phi$ direction by introducing the $2 \mathrm{~d}$ gauge field $B, \bar{B}[28$ we get

$$
\begin{gathered}
L=R^{2}(\partial \varphi+B)(\bar{\partial} \varphi+\bar{B})-\partial t \bar{\partial} t+2 A_{i}(x) \bar{\partial} x^{i}[R(\partial \varphi+B)-\partial t] \\
+\partial x^{i} \bar{\partial} x^{i}+\alpha^{\prime}(B \bar{\partial} \tilde{\varphi}-\bar{B} \partial \tilde{\varphi})
\end{gathered}
$$

where $\phi=R \varphi, 0<\varphi \leq 2 \pi$ and $\tilde{\varphi}$ is the Lagrange multiplier that sets the field strength of $B, \bar{B}$ to zero. Fixing the gauge $\varphi=0$ and integrating over $B, \bar{B}$ we get the dual Lagrangian

$$
\begin{gathered}
\tilde{L}=\tilde{R}^{2} \partial \tilde{\varphi} \bar{\partial} \tilde{\varphi}-\partial t \bar{\partial} t+2 A_{i}(x) \bar{\partial} x^{i}(\tilde{R} \partial \tilde{\varphi}-\partial t)+\partial x^{i} \bar{\partial} x^{i} \\
=\partial \tilde{u} \bar{\partial} \tilde{v}+2 A_{i}(x) \bar{\partial} x^{i} \partial \tilde{u}+\partial x^{i} \bar{\partial} x^{i}
\end{gathered}
$$

where $\tilde{R} \equiv \alpha^{\prime} / R, \tilde{u}=\tilde{R} \tilde{\varphi}-t, \tilde{v}=\tilde{R} \tilde{\varphi}+t$. Thus the theory (2.5) is invariant under $\varphi \rightarrow \tilde{\varphi}$, $R \rightarrow \tilde{R}$ with fixed $A_{i}$

The $F_{i j}=$ const model (2.13) has also rotational symmetry in each of the 2-planes. For example, in the $D=4$ model (2.14) we can thus make duality rotation in the $\theta$ direction. Gauging $\theta \rightarrow \theta+a$ symmetry get the following model

$$
L=\partial u \bar{\partial} v+\partial r \bar{\partial} r+r^{2}(\partial \theta+B)(\bar{\partial} \theta+\bar{B})+f r^{2} \partial u(\bar{\partial} \theta+\bar{B})+\alpha^{\prime}(B \bar{\partial} \tilde{\theta}-\bar{B} \partial \tilde{\theta}),
$$

where $\tilde{\theta}$ is the Lagrange multiplier (dual coordinate) and $B, \bar{B}$ are components of the $2 \mathrm{~d}$ gauge field. Making field redefinition (see also [12]) $B=B^{\prime}-f \partial u, v=v^{\prime}+\alpha^{\prime} f \tilde{\theta}$ we can transform the model (2.31) into the flat space one.15 This transformation is not, however, allowed in general since it is not globally well defined. The dual coordinate $\tilde{\theta}$ must have period $2 \pi$ [28], so that $v^{\prime}$ is defined only if both $\phi=(v+u) / 2$ and $t=(v-u) / 2$ are assumed to be compact with period $2 \pi R$, and if $f, R$ satisfy $\alpha^{\prime} f=2 k R, k=$ integer.

14 It is possible to describe the theory using the manifestly duality invariant "doubled" action [29] in which both the original and dual fields $\varphi$ and $\tilde{\varphi}$ are present. Starting with (2.29), fixing the non-Lorentz-covariant gauge $B_{1}=0$ and integrating over the $B_{0}$ component $\left(B \rightarrow B_{1}-B_{0}, \bar{B} \rightarrow\right.$ $\left.B_{1}+B_{0}\right)$ one finds $L_{\text {doubl. }}=-\frac{1}{2} \dot{\varphi} \tilde{\varphi}^{\prime}+\frac{1}{4} R^{2} \varphi^{\prime 2}+\frac{1}{4} \tilde{R}^{2} \tilde{\varphi}^{\prime 2}+A_{-}\left(R \varphi^{\prime}+\tilde{R} \tilde{\varphi}^{\prime}\right)+A_{-} A_{-}+O\left(t, x^{i}\right)$, where prime is $\partial_{\sigma}$, dot is $\partial_{\tau}$ and $A_{-}=A_{i}(x) \partial_{-} x^{i} . L_{\text {doubl. }}$ can be interpreted as a Lagrangian for $\varphi$ written in terms of the coordinates and momenta (represented by $\tilde{\varphi}^{\prime}$ ), so that its $\dot{\varphi}$-independent part is proportional to the Hamiltonian. It is obviously symmetric under $\varphi \rightarrow \tilde{\varphi}, R \rightarrow \tilde{R}=\alpha^{\prime} / R$. If one integrates out $\tilde{\varphi}^{\prime}$ (or $\varphi$ ) one gets back to the original (2.5) (or dual (2.30) ) action (for related canonical approach to duality see [30]).

15 The original $\sigma$-model is thus related to a flat space one by a combination of duality, coordinate transformation and "inverse" duality (cf. [31,18]). If, however, the true starting point is the "doubled" or "gauged' model (2.31), then the transformation to the model corresponding to the flat space is just a coordinate transformation on the extended configuration space of $(u, v, r, \theta, \tilde{\theta}, B, \bar{B})$. 


\subsection{Point-particle limit and solution of tachyon equation}

The basic differential operator which dictates the propagation of point-like (ground state) scalar particles in the background (2.5) and which should be the zero-mode part of the Hamiltonian of the corresponding CFT is, to leading order in the $\alpha^{\prime}$-expansion, the Laplacian of the metric (2.8) (the dilaton here is constant). It has the simple form

$$
\Delta=-\frac{1}{\sqrt{-G}} \partial_{\mu}\left(\sqrt{-G} G^{\mu \nu} \partial_{\nu}\right)=-4 \partial_{u} \partial_{v}-\left[\partial^{i}-2 A^{i}(x) \partial_{v}\right]\left[\partial_{i}-2 A_{i}(x) \partial_{v}\right]
$$

The exact tachyon vertex operator is thus the solution of

$$
\alpha^{\prime} \Delta T+O\left(\alpha^{2}\right) T=4 T
$$

The $O\left(\alpha^{\prime 2}\right)$ corrections are scheme-dependent and may be non-vanishing in the scheme in which the exact expressions for the $\sigma$-model couplings (metric, dilaton and antisymmetric tensor) do not depend on $\alpha^{\prime}$ (for a discussion of $\alpha^{\prime}$-corrections to the tachyon equation see [56] and refs. there). The exact form of the tachyon equation is usually hard to determine without knowledge of the underlying conformal theory. It turns out that in the present model one can actually fix its form already at the $\sigma$-model level by using the relation (for $R \rightarrow \infty)$ to the WZW model of ref. [17]. Let us first omit the $\alpha^{\prime}$-corrections in (2.33) (we shall discuss how to incorporate them at the end of this subsection).

To relate the operator (2.32) to the Hamiltonian of a point-particle limit of our model let us ignore the $\sigma$-dependence of the fields in (2.5) $(v, u=\phi \pm t)$

$$
I_{\text {particle }}=\frac{1}{4 \alpha^{\prime}} \int d \tau\left[\dot{u} \dot{v}+\dot{x}^{i} \dot{x}_{i}+2 A_{i}(x) \dot{x}^{i} \dot{u}\right] .
$$

On the equations of motion $u=u_{0}+p_{v} \tau, \dot{u}=p_{v}=$ const, so that (2.34) becomes very similar to the standard action $\left(\int d \tau\left[-\dot{t}^{2}+\dot{x}^{i} \dot{x}_{i}+e A_{i}(x) \dot{x}^{i}\right]\right)$ of a charged particle in an external magnetic field. The peculiarity of the present model is that the charge $e$ is equal to (twice) the "light-cone" momentum $p_{v}$, or, equivalently, to the sum of the momentum in the compact direction (or "Kaluza-Klein charge") $p_{\phi}$ and the energy $p_{t}=E, p_{v}=\frac{1}{2}\left(p_{\phi}+E\right)$. The dependence on $E$ is due to the curvature of space-time (proportional to the magnetic field energy density, cf. (2.27)). The Hamiltonian corresponding to (2.34)

$$
H=4 \alpha^{\prime}\left\{p_{u} p_{v}+\frac{1}{4}\left[p_{i}-2 p_{v} A_{i}(x)\right]^{2}\right\}
$$

directly leads to (2.32) upon quantisation. Representing the solution of (2.33) as (if $\phi$ is periodic the integral over $p_{u}+p_{v}$ is actually a sum)

$$
T(u, v, x)=\int d p_{u} d p_{v} \exp \left(i p_{u} u+i p_{v} v\right) \tilde{T}\left(p_{u}, p_{v}, x\right)
$$


we find that $\tilde{T}$ satisfies

$$
\begin{gathered}
-\left[\partial^{i}-i e A^{i}(x)\right]\left[\partial_{i}-i e A_{i}(x)\right] \tilde{T}=\mu \tilde{T}, \\
e \equiv 2 p_{v}=p_{\phi}+E, \quad \mu=4 / \alpha^{\prime}-4 p_{u} p_{v} .
\end{gathered}
$$

This equation is formally the same as the Schrödinger equation for a charged particle in a magnetic field.16

Eq. (2.37) can be easily solved explicitly for the constant magnetic field (2.12), for example, in the $D=4$ case of $F_{i j}=f \epsilon_{i j}$. Using the gauge (2.12), i.e. $A_{i}=-\frac{1}{2} f \epsilon_{i j} x^{j}$ one can put (2.37) in the form (we assume $f p_{v}>0$ )

$$
\begin{gathered}
2\left(C^{\dagger} C+C C^{\dagger}\right) \tilde{T}=\mu \tilde{T} \\
C^{\dagger} \equiv-i\left(\partial_{x}-\frac{1}{2} f p_{v} x^{*}\right), \quad C \equiv-i\left(\partial_{x}^{*}+\frac{1}{2} f p_{v} x\right), \quad x \equiv x_{1}+i x_{2}, \quad x^{*} \equiv x_{1}-i x_{2}, \\
{\left[C, C^{\dagger}\right]=f p_{v}, \quad\left[b_{0}, b_{0}^{\dagger}\right]=1, \quad C \equiv \sqrt{f p_{v}} b_{0} .}
\end{gathered}
$$

Thus the spectrum is given by

$$
4 f p_{v}\left(l+\frac{1}{2}\right)=\mu, \quad l=0,1,2, \ldots .
$$

Defining also $B^{\dagger} \equiv-i\left(\partial_{x}^{*}-\frac{1}{2} f p_{v} x\right), \quad B \equiv-i\left(\partial_{x}+\frac{1}{2} f p_{v} x^{*}\right),\left[B, B^{\dagger}\right]=f p_{v}$ (so that the angular momentum $J \sim B^{\dagger} B-C^{\dagger} C$ ) one can represent the eigen-functions as: $\psi_{l, n}=$ $a_{l, n}\left(B^{\dagger}\right)^{n}\left(C^{\dagger}\right)^{l} \exp \left(-\frac{1}{2} f p_{v}|x|^{2}\right) .17$

Alternatively, we may follow Landau [33] and solve (2.37) in the gauge $A_{1}=$ $-f x_{2}, A_{2}=0$. According to (2.6) this gauge is related to (2.12) used above by a shift of $v$ so that $v$ in the tachyon vertex (2.36) will be related to $v$ in (2.14) by $v \rightarrow v+f x_{1} x_{2}$. After setting

$$
\tilde{T}=\int d p_{1} e^{i p_{1} x_{1}} \mathcal{T}\left(p_{u}, p_{v}, p_{1}, x_{2}\right)
$$

16 One should keep in mind, of course, that the original equation we started with (2.32) is not a Klein-Gordon equation for a charged particle in flat space, since it contains an extra $\partial_{t}$-term (in $\partial_{v}$ ) on the usual place of the charge. This is reflected in the presence of the energy in the above relation for the effective charge $e$.

17 The final expression for $T$ may have a simple integral representation in terms of exponent of a bilinear form in $x$. This is suggested by the observation that in the case when $\phi$ is noncompact we can use transformation (2.15) to put the action into the plane-wave type form (2.16). For the plane wave background (2.17) the Klein-Gordon equation is solved by [32] $T\left(u, v^{\prime}, y\right)=$ $\int d p_{v} d p_{i} \hat{T}\left(p_{v}, p_{i}\right) \exp \left[i p_{v} v^{\prime}+i\left(\alpha^{\prime} p_{v}\right)^{-1} u+i p_{i} y^{i}-\frac{1}{4} i p_{v}^{-1} \int d u g^{i j}(u) p_{i} p_{j}\right]$. In our particular case of (2.16), $\int d u g^{i j}(u) p_{i} p_{j}=(\sin u)^{-1}\left[\cos u\left(p_{1}^{2}+p_{2}^{2}\right)-2 p_{1} p_{2}\right]$, and $y^{i}$ are related to the original coordinates $x^{i}$ by the linear transformation (2.15). 
eq. (2.37) becomes the standard harmonic oscillator differential equation,

$$
\left[-\partial_{2}^{2}+\omega^{2}\left(x_{2}-b\right)^{2}\right] \mathcal{T}=\mu \mathcal{T}, \quad \omega \equiv 2 p_{v} f, \quad b \equiv-p_{1} / \omega,
$$

with the solution

$$
\mathcal{T}\left(p_{u}, p_{v}, p_{1}, x_{2}\right)=\sum_{l=0}^{\infty} c_{l}\left(p_{u}, p_{v}, p_{1}\right) \exp \left[-\frac{1}{2} \omega\left(x_{2}-b\right)^{2}\right] H_{l}\left[\sqrt{\omega}\left(x_{2}-b\right)\right],
$$

where $\mu$ (i.e. $p_{u}, p_{v}$, see (2.37)) and $l$ are related by the quantization condition equivalent to 2.41)

$$
\mu=2 \omega\left(l+\frac{1}{2}\right), \quad 4 / \alpha^{\prime}-4 p_{u} p_{v}=4 p_{v} f\left(l+\frac{1}{2}\right) .
$$

Setting $p_{v}=\frac{1}{2}\left(p_{\phi}+E\right)_{\text {. }} p_{u}=\frac{1}{2}\left(p_{\phi}-E\right)$, where $p_{\phi}=m / R, m=0, \pm 1, \pm 2, \ldots$ if $\phi$ is compactified on a circle 18 we obtain the formula for the tachyon energy 19

$$
-E^{2}+p_{\phi}^{2}+2\left(p_{\phi}+E\right) f\left(l+\frac{1}{2}\right)=4 / \alpha^{\prime} .
$$

As we shall see in Section 3.4 the expressions $(2.41),(2.46)$ for the tachyon spectrum agree with the result which follows from the corresponding conformal theory up to just one $\alpha^{\prime}$ correction term. It is possible to understand the origin of this correction term as follows. In general, the differential operator in (2.33) contains corrections of the form $\left(G^{\mu \nu}+\right.$ $\left.c \alpha^{\prime} H^{\mu \lambda \rho} H_{\lambda \rho}^{\nu}+\ldots\right) D_{\mu} D_{\nu}+\ldots$ In the present model the non-vanishing component of $H_{\mu \nu \lambda}$ is $H_{u i j}=-f \epsilon_{i j}$ and using its 'null' structure it is possible to argue that all other corrections are absent (assuming one uses the scheme based on dimensional regularisation). Comparison with WZW $\sigma$-model shows that in the scheme where the metric does not receive $\alpha^{\prime}$-corrections $c=-1 / 4$ [56]. Alternatively, in the "CFT scheme" where the tachyon equation is not modified by $\alpha^{\prime}$-corrections the exact expression for the metric is given by

$$
G_{\mu \nu}^{\prime}=G_{\mu \nu}-c \alpha^{\prime} H_{\mu \lambda \rho} H_{\nu}^{\lambda \rho}=G_{\mu \nu}+\frac{1}{2} \alpha^{\prime} f^{2} \delta_{\mu u} \delta_{\nu u}
$$

so that the exact $\sigma$-model action receives an extra term $\frac{1}{2} \alpha^{\prime} f^{2} \partial u \bar{\partial} u$ (and thus (2.34) also gets the correction $\frac{1}{2} \alpha^{\prime} f^{2} \dot{u} \dot{u}$ ). The presence of such term is in agreement with the expression for the stress tensor (or Hamiltonian) in the $E_{2}^{c}$ WZW theory [18,20] (because of the null structure of the $\alpha^{\prime}$ correction term in the stress tensor its presence is consistent with the "free" value of the central charge). While in the $E_{2}^{c}$ WZW model the $\alpha^{\prime} \partial u \bar{\partial} u$ term in the action can be redefined away by a shift of $v$ this is no longer possible in the present model where $u+v$ is compact. Irrespective of the interpretation (i.e. of the scheme choice) the tachyon equation thus contains an extra $2 \alpha^{\prime} f^{2} \partial_{v}^{2}$ term, i.e. $\mu$ in $(2.37),(2.38)$ should be shifted by $2 \alpha^{\prime} f^{2} p_{v}^{2}$. As a result, the $\alpha^{\prime}$-corrected form of (2.46) becomes equivalent to the CFT expression.

Combining (2.36), (2.42), (2.44) and performing the integral over $E=p_{v}-p_{u}$ using (2.46) we finish with the expression for the general solution of (2.36) $T$ in terms of the integral over $p_{1}$ and the double sum over $l$ and $m$. The tachyon vertex operator with given conserved quantum numbers corresponds to a particular term in this representation.

18 Note that if $\phi$ were not periodic we could eliminate the dependence on $f$ by absorbing it into a rescaling of $E$ and $p_{\phi}, p_{\phi}+E \rightarrow f^{-1}\left(p_{\phi}+E\right), p_{\phi}-E \rightarrow f\left(p_{\phi}-E\right)$.

19 Represented in terms of the dimensionless parameters this equation becomes $-E^{\prime 2}+m^{2}+$ $(2 l+1)\left(m+E^{\prime}\right) h=4 r^{2}, \quad E^{\prime} \equiv R E, \quad h \equiv f R, \quad r \equiv R / \sqrt{\alpha^{\prime}}$. 


\section{Light-cone gauge quantisation and spectrum}

In this section we shall solve the classical string equations corresponding to the $D=4$ theory (2.14) and then quantise the model in the light-cone gauge. This is possible to do explicitly for the model (2.13) in any $D$ due to its special structure: (i) for all the models (2.5) there are the infinite dimensional symmetries $u^{\prime}=u+h(\tau-\sigma), v^{\prime}=v+g(\tau+\sigma)$, implying the existence of the two conserved chiral currents $\mathcal{J}_{+}=\partial_{+} u, \mathcal{J}_{-}=\partial_{-} v+$ $2 A_{i}(x) \partial_{-} x^{i}$; (ii) the Lagrangian (2.13) or (2.14) is bilinear in $x^{i}$. The existence of the light-cone gauge makes the unitary of the model explicit.

\subsection{Solution of the classical string equations}

Using Minkowski world sheet notation and introducing the complex coordinates $x=$ $x^{1}+i x^{2}, x^{*}=x^{1}-i x^{2}$ one can represent the $D=4$ Lagrangian (2.14) in the following form:

$$
L=\partial_{+} u \partial_{-} v+\partial_{+} x \partial_{-} x^{*}+\frac{1}{2} i f \partial_{+} u\left(x \partial_{-} x^{*}-x^{*} \partial_{-} x\right)+\partial_{+} x^{a} \partial_{-} x^{a},
$$

where we have added $D_{\text {tot }}-4$ free scalar fields $x^{a}\left(a=3, \ldots, D_{t o t}-1\right)$ which are needed to saturate the central charge condition. It is easy to show 12 that the model (2.5), (2.7) has the same central charge as the free one, so we are to choose $D_{t o t}=26$. The free-field contribution of $x^{a}$ will be ignored in the most of our discussion. The equations of motion are then given by

$$
\begin{gathered}
\partial_{-} \partial_{+} u=0, \quad \partial_{+}\left[\partial_{-} v+\frac{1}{2} i f\left(x \partial_{-} x^{*}-x^{*} \partial_{-} x\right)\right]=0 \\
\partial_{+} \partial_{-} x^{*}=i f \partial_{+} u \partial_{-} x^{*}, \quad \partial_{+} \partial_{-} x=-i f \partial_{+} u \partial_{-} x .
\end{gathered}
$$

Their general solution is

$$
\begin{gathered}
u=u_{+}+u_{-}, \quad x=\exp \left(-i f u_{+}\right) y, \quad x^{*}=\exp \left(i f u_{+}\right) y^{*}, \quad y=y_{+}+y_{-}, \\
v=v_{+}+v_{-}+\frac{1}{2} i f\left(y_{+}^{*} y_{-}-y_{-}^{*} y_{+}\right) \\
\phi=\frac{1}{2}(u+v)=\phi_{+}+\phi_{-}+\frac{1}{4} i f\left(y_{+}^{*} y_{-}-y_{-}^{*} y_{+}\right)
\end{gathered}
$$

where $u$ and $y$ satisfy the free wave equations and lower signs indicate dependence on $\sigma_{+}=\sigma+\tau$ or $\sigma_{-}=\tau-\sigma$, i.e. $u_{ \pm}=u_{ \pm}\left(\sigma_{ \pm}\right), v_{ \pm}=v_{ \pm}\left(\sigma_{ \pm}\right)$, etc. Thus $x, x^{*}$ are related to the free fields $y, y^{*}$ by an $S O(2)$ rotation.

Let us now fix the residual conformal symmetry $\sigma_{ \pm} \rightarrow F\left(\sigma_{ \pm}\right)$by choosing the "lightcone" gauge:

$$
u=u_{0}+p_{+} \sigma_{+}+p_{-} \sigma_{-} .
$$

Then $x, x^{*}$ can be written as ( $u_{0}$ is absorbed into the definition of $y$ )

$$
x=\exp \left(-i f p_{+} \sigma_{+}\right) y, \quad x^{*}=\exp \left(i f p_{+} \sigma_{+}\right) y^{*} .
$$


The periodicity condition $x(\sigma+\pi, \tau)=x(\sigma, \tau)$ is solved if $y$ satisfies the "twisted" boundary condition,

$$
y(\sigma+\pi, \tau)=\exp \left(i f p_{+} \pi\right) y(\sigma, \tau)
$$

implying

$$
y_{+}=\exp \left(i f p_{+} \sigma_{+}\right) z_{+}, \quad y_{-}=\exp \left(-i f p_{+} \sigma_{-}\right) z_{-},
$$

where $z_{ \pm}$can be expanded as follows,

$$
z_{+}=i \sqrt{\alpha^{\prime} / 2} \sum_{n} \tilde{a}_{n} \exp \left(-2 i n \sigma_{+}\right), \quad z_{-}=i \sqrt{\alpha^{\prime} / 2} \sum_{n} a_{n} \exp \left(-2 i n \sigma_{-}\right)
$$

The explicit form of the solution is thus

$$
\begin{gathered}
x=z_{+}+\exp \left(-2 \tau i f p_{+}\right) z_{-}, \quad x^{*}=z_{+}^{*}+\exp \left(2 \tau i f p_{+}\right) z_{-}^{*}, \\
v=v_{+}+v_{-}+\frac{1}{2} i f\left[\exp \left(-2 i f p_{+} \tau\right) z_{+}^{*} z_{-}-\exp \left(2 i f p_{+} \tau\right) z_{-}^{*} z_{+}\right], \\
\phi=\frac{1}{2}(u+v)=\phi_{+}+\phi_{-}+\frac{1}{4} i f\left[\exp \left(-2 i f p_{+} \tau\right) z_{+}^{*} z_{-}-\exp \left(2 i f p_{+} \tau\right) z_{-}^{*} z_{+}\right] .
\end{gathered}
$$

Since $\phi$ is assumed to be periodic with period $2 \pi R$, its zero mode part will include the winding term. The zero mode parts of the fields are then

$$
\begin{gathered}
\phi_{\text {zero }}=\phi_{0}+q \sigma+s \tau, \quad t_{\text {zero }}=t_{0}+p \tau, \quad q=2 R w, \\
u_{\text {zero }}=u=u_{0}+p_{+} \sigma_{+}+p_{-} \sigma_{-}, \quad p_{ \pm}=\frac{1}{2}( \pm q+s-p), \\
v_{\text {zero }}=v_{0}+q_{+} \sigma_{+}+q_{-} \sigma_{-}, \quad q_{ \pm}=\frac{1}{2}( \pm q+s+p) .
\end{gathered}
$$

Here $w$ is an integer winding number and $s$ and $p$ take continuous values.

Above we assumed that momentum $p_{+}$has generic value. A special case is when $f p_{+}=2 k, k=0, \pm 1, \pm 2, \ldots$. In the sector with such special $p_{+}$the field $y(\sigma, \tau)$ satisfies the standard "untwisted" boundary condition and thus contains the translational part $p_{y} \tau$ (absent for generic $f p_{+}$). That means that for such $f p_{+}$(i.e. for special $p_{+}$for a given $f$ or for special magnetic field in each $p_{+}$-sector) the " $y$-string" can move freely on the 2-plane. The solution corresponding to this special case can still be described by (3.11) if we rescale the oscillators $\tilde{a}_{n}=\left(n-\frac{1}{2} f p_{+}\right)^{-1} \tilde{a}_{n}^{\prime}, \quad a_{n}=\left(n+\frac{1}{2} f p_{+}\right)^{-1} a_{n}^{\prime}$ and take the limit $f p_{+}=2 k+\epsilon, \epsilon \rightarrow 0$ (so that $\tilde{a}_{k}^{\prime}+a_{-k}^{\prime} \sim p_{y}$ ).20

20 The solution for $y$ is analogous to string motion on the $R^{2} / Z_{k}$ orbifold [34] with generic solution representing string modes of the twisted sector (localised near the tip of the cone) and the special one - the untwisted sector (strings that can move over the plane). 


\subsection{Stress tensor and Hamiltonian}

The non-vanishing stress tensor components corresponding to the model (2.5) are given by

$$
T_{ \pm \pm}=\partial_{ \pm} u \partial_{ \pm} v+2 A_{i}(x) \partial_{ \pm} x^{i} \partial_{ \pm} u+\partial_{ \pm} x^{i} \partial_{ \pm} x_{i}
$$

Computed on the classical solution in the present case of $(3.1),(3.4)$ they are

$$
\begin{aligned}
& T_{--}=p_{-} \partial_{-} v_{-}+\partial_{-} y_{-} \partial_{-} y_{-}^{*}+\frac{1}{2} i f p_{-}\left(y_{-} \partial_{-} y_{-}^{*}-y_{-}^{*} \partial_{-} y_{-}\right) \\
& T_{++}=p_{+} \partial_{+} v_{+}+\partial_{+} y_{+} \partial_{+} y_{+}^{*}-\frac{1}{2} i f p_{+}\left(y_{+} \partial_{+} y_{+}^{*}-y_{+}^{*} \partial_{+} y_{+}\right) .
\end{aligned}
$$

The classical constraints $T_{--}=T_{++}=0$ are then easily solved and, as usual, determine $v_{ \pm}$in terms of the free fields $y_{ \pm}$or $z_{ \pm}$. The classical expressions for the Virasoro operators $L_{0}, \tilde{L}_{0}$ are

$$
\begin{gathered}
L_{0}=\frac{1}{4 \pi \alpha^{\prime}} \int_{0}^{\pi} d \sigma T_{--}=\frac{p_{-} q_{-}}{4 \alpha^{\prime}}+\frac{1}{2} \sum_{n}\left(n+\frac{1}{2} f p_{+}\right)\left(n+\frac{1}{2} f q\right) a_{n}^{*} a_{n}, \\
\tilde{L}_{0}=\frac{1}{4 \pi \alpha^{\prime}} \int_{0}^{\pi} d \sigma T_{++}=\frac{p_{+} q_{+}}{4 \alpha^{\prime}}+\frac{1}{2} \sum_{n} n\left(n-\frac{1}{2} f p_{+}\right) \tilde{a}_{n}^{*} \tilde{a}_{n} .
\end{gathered}
$$

Hence the Hamiltonian $H=L_{0}+\tilde{L}_{0}$ is given by

$$
H=\frac{q^{2}+s^{2}-p^{2}}{8 \alpha^{\prime}}+\frac{1}{2} \sum_{n}\left(n+\frac{1}{2} f p_{+}\right)\left(n+\frac{1}{2} f q\right) a_{n}^{*} a_{n}+\frac{1}{2} \sum_{n} n\left(n-\frac{1}{2} f p_{+}\right) \tilde{a}_{n}^{*} \tilde{a}_{n} .
$$

The relation to the point-particle expression (2.35) is established by dropping all $\sigma$ dependence (i.e. terms with $n \neq 0$ ), setting winding number $q=0$ and expressing $s$ and $p$ in terms of conserved momenta $p_{u}$ and $p_{v}$.

\subsection{Operator quantisation}

We can now quantise the theory in a standard way by promoting the Fourier modes to operators acting in a Fock space and demanding the canonical commutation relations,

$$
\left[P(\sigma), x^{*}\left(\sigma^{\prime}\right)\right]=\left[P^{*}(\sigma), x\left(\sigma^{\prime}\right)\right]=-i \delta\left(\sigma-\sigma^{\prime}\right), \quad\left[x^{i}\left(\sigma^{\prime}\right), \partial_{\sigma} x^{j}(\sigma)\right]=0
$$

where $P=\frac{1}{2}\left(P_{1}+i P_{2}\right), P^{*}=\frac{1}{2}\left(P_{1}-i P_{2}\right)$ are the momenta. Using that $P=\frac{1}{4 \pi \alpha^{\prime}}\left(\partial_{\tau} x+\right.$ if $\left.p_{+} x\right)$ we find the relations

$$
\left[a_{n}, a_{m}^{*}\right]=2\left(n+\frac{1}{2} f p_{+}\right)^{-1} \delta_{n m}, \quad\left[\tilde{a}_{n}, \tilde{a}_{m}^{*}\right]=2\left(n-\frac{1}{2} f p_{+}\right)^{-1} \delta_{n m} .
$$


We can thus introduce the creation and annihilation operators:

$$
\begin{gathered}
{\left[b_{n \pm}, b_{m \pm}^{\dagger}\right]=\delta_{n m}, \quad\left[\tilde{b}_{n \pm}, \tilde{b}_{m \pm}^{\dagger}\right]=\delta_{n m}, \quad\left[b_{0}, b_{0}^{\dagger}\right]=1, \quad\left[\tilde{b}_{0}, \tilde{b}_{0}^{\dagger}\right]=1,} \\
b_{n+}^{\dagger}=a_{-n} \omega_{-}, \quad b_{n+}=a_{-n}^{*} \omega_{-}, \quad b_{n-}^{\dagger}=a_{n}^{*} \omega_{+}, \quad b_{n-}=a_{n} \omega_{+}, \\
\tilde{b}_{n+}^{\dagger}=\tilde{a}_{-n} \omega_{+}, \quad \tilde{b}_{n+}=\tilde{a}_{-n}^{*} \omega_{+}, \quad \tilde{b}_{n-}^{\dagger}=\tilde{a}_{n}^{*} \omega_{-}, \quad \tilde{b}_{n-}=\tilde{a}_{n} \omega_{-}, \\
b_{0}^{\dagger}=\frac{1}{2} \sqrt{f p_{+}} a_{0}^{*}, \quad b_{0}=\frac{1}{2} \sqrt{f p_{+}} a_{0}, \quad \tilde{b}_{0}^{\dagger}=\frac{1}{2} \sqrt{f p_{+}} \tilde{a}_{0}, \quad \tilde{b}_{0}=\frac{1}{2} \sqrt{f p_{+}} \tilde{a}_{0}^{*}
\end{gathered}
$$

where $\omega_{ \pm} \equiv \sqrt{\frac{1}{2}\left(n \pm \frac{1}{2} f p_{+}\right)}, \quad n=1,2, \ldots$. The subindices \pm correspond to components with spin "up" and "down" respectively. Above we have assumed $0<f p_{+}<2$. For $f p_{+}>2$ or $f p_{+}<0$ the creation/annihilation roles of some operators are changed and the analysis remains essentially the same (see [57] for a detailed discussion of this point).

In what follows we shall assume that all operators act on a particular eigen-state of the zero-mode operators so that the latter $(q, s, p$, etc.) can be replaced by their eigen-values. The operators $L_{0}$ and $\tilde{L}_{0}$ will be normal ordered with the ordering constant being fixed by the Virasoro algebra. The free-theory normal ordering constant is shifted from 1 to $1-\frac{1}{4} \gamma\left(1-\frac{1}{2} \gamma\right)$ where $\gamma \equiv f p_{+}$. This corresponds to the use of the generalised $\zeta$-function regularisation (see also [57])

$$
\sum_{n=1}^{\infty}(n+c)=\lim _{s \rightarrow-1} \sum_{n=1}^{\infty}(n+c)^{-s}=-\frac{1}{12}+\frac{1}{2} c(1-c) .
$$

Similar result is found in the open string theory in a constant magnetic field [3] and is typical to the case of a free scalar field with twisted boundary conditions. We will see that this shift is also consistent with the modular invariant path integral approach discussed in Section 4.

Given that $\phi$ is a compact coordinate, the zero mode part of its canonical momentum should be quantized $\left(\left[p_{\phi}, \phi_{0}\right]=-i\right)$,

$$
p_{\phi}=\int_{0}^{\pi} d \sigma P_{\phi}=\frac{m}{R}, \quad m=0, \pm 1, \pm 2, \ldots .
$$

On the other hand, using eqs. (3.13), (3.11), (3.10), we obtain

$$
p_{\phi}=\frac{1}{2 \pi \alpha^{\prime}} \int_{0}^{\pi} d \sigma\left[\partial_{\tau} \phi+\frac{1}{2} i f\left(x \partial_{-} x^{*}-x^{*} \partial_{-} x\right)\right]=\frac{1}{2} \alpha^{\prime-1} s+f \hat{J}_{R} .
$$

Here $\hat{J}_{R}$ is the "right" part of the angular momentum operator, which after symmetrising the classical expression and normal ordering has the form

$$
\hat{J}_{R}=-b_{0}^{\dagger} b_{0}-\frac{1}{2}+\sum_{n=1}^{\infty}\left(b_{n+}^{\dagger} b_{n+}-b_{n-}^{\dagger} b_{n-}\right)=J_{R}-\frac{1}{2}
$$


Since our background is stationary, the string has also conserved energy operator

$$
E=\int_{0}^{\pi} d \sigma P_{t}=-\frac{1}{2 \pi \alpha^{\prime}} \int_{0}^{\pi} d \sigma\left[\partial_{\tau} t+\frac{1}{2} i f\left(x \partial_{-} x^{*}-x^{*} \partial_{-} x\right)\right]=-\frac{1}{2} \alpha^{\prime-1} p-f \hat{J}_{R} .
$$

Inserting eqs. (3.26), (3.27), (3.30), (3.32) in eqs. (3.20), (3.21) and (3.22) we finally obtain (we include also the contribution of the extra $D_{t o t}-4=22$ degrees of freedom $x^{a}$ )

$$
\begin{gathered}
\hat{L}_{0}=\frac{1}{4}\left[\alpha^{\prime}\left(-E^{2}+p_{a}^{2}\right)+\left(\frac{m}{r}-w r\right)^{2}\right]-\frac{1}{2} \alpha^{\prime}(Q+E) f J_{R}+N-c_{0}=L_{0}-c_{0}, \\
\hat{\tilde{L}}_{0}=\frac{1}{4}\left[\alpha^{\prime}\left(-E^{2}+p_{a}^{2}\right)+\left(\frac{m}{r}+w r\right)^{2}\right]-\frac{1}{2} \alpha^{\prime}(Q+E) f J_{R}+\tilde{N}-c_{0}=\tilde{L}_{0}-c_{0}, \\
\hat{H}=\frac{1}{2}\left[\alpha^{\prime}\left(-E^{2}+p_{a}^{2}\right)+w^{2} r^{2}+\frac{m^{2}}{r^{2}}\right]-\alpha^{\prime}(Q+E) f J_{R}+N+\tilde{N}-2 c_{0}=H-2 c_{0},
\end{gathered}
$$

with the Virasoro conditions $L_{0}=\tilde{L}_{0}=c_{0}$, where

$$
\begin{gathered}
r \equiv \frac{R}{\sqrt{\alpha^{\prime}}}, \quad Q \equiv \frac{1}{\sqrt{\alpha^{\prime}}}\left(\frac{m}{r}+w r\right)=p_{\phi}+\frac{w R}{\alpha^{\prime}}, \quad c_{0} \equiv 1-\frac{1}{4} \gamma\left(1-\frac{1}{2} \gamma\right), \\
\gamma \equiv f p_{+}=\alpha^{\prime}(Q+E) f
\end{gathered}
$$

and $N$ and $\tilde{N}$ are the operators

$$
\begin{aligned}
& N=\sum_{n=1}^{\infty} n\left(b_{n+}^{\dagger} b_{n+}+b_{n-}^{\dagger} b_{n-}+a_{n a}^{\dagger} a_{n a}\right), \\
& \tilde{N}=\sum_{n=1}^{\infty} n\left(\tilde{b}_{n+}^{\dagger} \tilde{b}_{n+}+\tilde{b}_{n-}^{\dagger} \tilde{b}_{n-}+\tilde{a}_{n a}^{\dagger} \tilde{a}_{n a}\right) .
\end{aligned}
$$

Note that $\hat{L}_{0}, \hat{\tilde{L}}_{0}, \hat{H}$ depend on non-trivially on $f$ because of the periodicity condition in $\phi$ : if $\phi$ were noncompact, $f$ could be eliminated from (3.1) by a coordinate transformation, or, equivalently, could be absorbed into continuous momenta and energy $\left(Q+E \rightarrow f^{-1}(Q+\right.$ $E), Q-E \rightarrow f(Q-E)$ ). The Hamiltonian (3.35) of our CFT contains one $\alpha^{\prime}$ correction term $\left(\gamma^{2}\right.$ in $\left.c_{0}\right)$. For $R=\infty$ it is in agreement with the Hamiltonian of the $E_{2}^{c}$ WZW theory which also contains one $1 / k$ correction [18]. This implies that the normal ordering (regularisation) prescription we have used corresponds to the normal ordering used in the current algebra approach.

The above operators and hence the spectrum are manifestly invariant under the duality transformation $m \leftrightarrow w, r \leftrightarrow 1 / r$. The field strength $f$ is coupled to a combination of the energy with the "left" momentum $Q$ in the $\phi$ direction as in the point-particle limit case discussed in Section 2.5. The linear term $E f J_{R}$ in $H$ coupling the energy to the magnetic field originates from the $A_{i}(x) \partial_{-} x^{i} \partial_{+} t$ term in the action (2.3) (which is necessary for 
conformal invariance and represents the deformation of the geometry due to the magnetic stress-energy density). Note also that only the right part of the angular momentum couples to the magnetic field (in particular, the zero mode operators corresponding to the left sector do not appear in the Hamiltonian).

The correspondence with the particle Hamiltonian 2.35, 2.38) is established by dropping all oscillator terms, i.e. keeping only the zero mode part of $\hat{J}_{R}$ in (3.31) (and setting $w=0)$,

$$
\hat{H}_{0}=\frac{1}{2} \alpha^{\prime}\left[-E^{2}+p_{\phi}^{2}+p_{a}^{2}+2\left(p_{\phi}+E\right) f\left(b_{0}^{\dagger} b_{0}+\frac{1}{2}\right)-\frac{1}{2} \alpha^{\prime} f^{2}\left(p_{\phi}+E\right)^{2}\right]-2,
$$

and observing that $b_{0}^{\dagger}, b_{0}$ directly correspond to the creation/annihilation operators in (2.40) (note that $p_{v}=\frac{1}{2}\left(p_{\phi}+E\right)$, i.e. $p_{v}$ corresponds to $\frac{1}{2} p_{+}$, cf. also $(3.11),(3.28)$ ). The zero mode part of $\hat{J}_{R}$ produces the Landau-type term in $H$ (3.35) (cf. 2.41)

$$
H_{\text {Landau }}=\alpha^{\prime}(Q+E) f\left(b_{0}^{\dagger} b_{0}+\frac{1}{2}\right) \rightarrow \alpha^{\prime}(Q+E) f\left(l+\frac{1}{2}\right), \quad l=0,1,2, \ldots,
$$

where $l$ represents the "right" part of the total orbital angular momentum.

The final Virasoro conditions are

$$
\begin{gathered}
L_{0}=\tilde{L}_{0} \rightarrow N-\tilde{N}=m w \\
H=2 c_{0} \rightarrow \kappa\left[E+\kappa^{-1} f\left(\hat{J}_{R}+\frac{1}{2} \alpha^{\prime} Q f\right)\right]^{2} \\
=-4 \alpha^{\prime-1}+4 \tilde{N} \alpha^{\prime-1}+\kappa^{-1}\left(Q-f \hat{J}_{R}\right)^{2}+p_{a}^{2} \\
\kappa \equiv 1+\frac{1}{2} \alpha^{\prime} f^{2} .
\end{gathered}
$$

Here $\hat{J}_{R}$ is the sum of the "right" contributions to the orbital and spin momentum,

$$
\hat{J}_{R}=-b_{0}^{\dagger} b_{0}-\frac{1}{2}+S_{R} \rightarrow-\left(l+\frac{1}{2}\right)+S_{R}, \quad S_{R}=\sum_{n=1}^{\infty}\left(b_{n+}^{\dagger} b_{n+}-b_{n-}^{\dagger} b_{n-}\right) .
$$

Eq. (3.42) implies the following expression for the magnetic dipole moment of an arbitrary physical state: $\mu=\sqrt{\alpha^{\prime}}(1+Q / M) S_{R}$, where $M$ is the rest mass of a state. Hence the tree-level gyromagnetic factor $g$ (defined in the frame corresponding to the stationary metric $(2.19))$ is given by

$$
g=2\left(1+\frac{M}{Q}\right) \frac{\left\langle S_{R}\right\rangle}{\langle S\rangle}
$$

where $S$ is the total spin (i.e. the sum of the left and right contributions). A similar formula (without the $M / Q$ term) was obtained in ref. [10] for the model (2.1) which does not contain the coupling $A_{i}(x) \partial_{-} x^{i} \partial_{+} t$ (the expression of 10 turns out to be more universal than (3.44), for a detailed discussion see [57]). We thus confirm the result of [10] that there may be physical states with $g \neq 2$. This is a novel feature of the closed string theory (all standard elementary particles have $g=2$ as the tree-level value of the gyromagnetic factor; $g=2$ is also true for the states of the open string theory in a magnetic field [35]). See also a discussion in Section 6. 


\subsection{Spectrum}

Physical states are constructed by applying the creation operators (i.e. $b_{n \pm}^{\dagger}, \tilde{b}_{n \pm}^{\dagger}$ and $\left.a_{n a}^{\dagger}, \quad \tilde{a}_{n a}^{\dagger}\right)$ to the Fock space vacuum $\left|0 ;\left\{m, w, l, p_{a}, E\right\}\right\rangle$, where $m$ and $w$ satisfy (3.41). Henceforth we choose the frame in which $p_{a}=0$. For $f=0$ the spectrum is the standard one of the bosonic closed string theory with one dimension compactified on $S^{1}$. For a non-zero field $f$ the energy levels split according to the value of the "right" contribution to the angular momentum $J_{R}$ and the "left" momentum in the compact direction $Q$.

The energy of the ground state $|0 ; m=w=l=0\rangle$ is shifted with respect to the zero-field case $\kappa E^{2}-E f=-4 / \alpha^{\prime}, \quad \kappa=1+\frac{1}{2} \alpha^{\prime} f^{2}$. It is always tachyonic. For the general scalar non-winding $(w=0)$ "tachyonic" state with nonzero $m$ and $l$ one has

$$
-E^{2}+Q^{2}+2(Q+E) f\left(l+\frac{1}{2}\right)-\frac{1}{2} \alpha^{\prime} f^{2}(Q+E)^{2}=4 / \alpha^{\prime}, \quad Q=p_{\phi}=m / R .
$$

This is the same expression as (2.46) we have found by directly solving the tachyon equation (2.33) in Section 2.5. Since $Q$ can take both positive and negative values there are tachyons for any $f$. At the same time, the energy levels of all the states with $Q=0$ are also modified by the presence of the magnetic field. As was already mentioned, the modification of the spectrum as compared to the zero-field case can be understood as being due to the presence of the curved metric and the antisymmetric tensor backgrounds induced by the magnetic field.

Let us now discuss some particular states. First, consider the zero-energy states with $N=\tilde{N}=1$. For example, a component of the "photon" with $S_{R} \neq 0$ is represented by $b_{1 \pm}^{\dagger} \tilde{a}_{1\left(D_{\text {tot }}-1\right)}^{\dagger}|0 ; m=w=0\rangle$. The "graviton" components correspond to $b_{1 \pm}^{\dagger} \tilde{b}_{1 \pm}^{\dagger}|0 ; m=w=0\rangle$ and $b_{1 \pm}^{\dagger} \tilde{a}_{1 a}^{\dagger}|0 ; m=w=0\rangle$.21 For these photon and graviton components one has $J_{R}= \pm 1-l$. Since $N=\tilde{N}=1$ one learns from eq. (3.42) that $E=0$ for any value of the Landau level $l$.

Next, let us look at the states which complete the $S U(2)_{R}$ massless vector multiplet in the $f=0$ theory compactified at the self-dual radius $r=1$. The components with $S_{R} \neq 0$ are given by $b_{1 \pm}^{\dagger}|0 ; m=w=1\rangle, \quad b_{1 \pm}^{\dagger}|0 ; m=w=-1\rangle$. For them $\tilde{N}=0, \quad J_{R}= \pm 1-l$, $l=0,1,2, \ldots$, and the energy

$$
\kappa\left[E+\kappa^{-1} f\left(\hat{J}_{R}+\frac{1}{2} \alpha^{\prime} Q f\right)\right]^{2}=-4 \alpha^{\prime-1}+\kappa^{-1}\left(Q-f \hat{J}_{R}\right)^{2},
$$

21 Note that with our choice of light-cone gauge on $u=\phi-t$ we have gauged away the oscillators of the compact "internal" coordinate $\phi$ instead of removing the oscillators of the longitudinal component $x^{D_{\text {tot }}-1}$. As a result, some of the states are represented in a way which is different from their usual light-cone form. 


$$
Q=\frac{1}{\sqrt{\alpha^{\prime}}}\left(r+r^{-1}\right)
$$

At the self-dual radius, $r=1$, an infinitesimal magnetic field $f>0$ renders the component with $J_{R}=1$ tachyonic. This instability is the same one as in the non-abelian gauge theory [36,37]. Away from the self-dual radius, this state has positive energy for small $f$ and becomes tachyonic at some critical magnetic field. The states corresponding to the $S U(2)_{L}$ multiplet have positive $E^{2}$ and $E^{2}=0$ at the self-dual radius (the state $b_{1 \pm}^{\dagger}|0 ; m=-w=-1\rangle$ for which $\tilde{N}=1$ and $J_{R}=-l$ has $E^{2}=0$ at $r=1$ regardless of the value of $f$ ).

The effect that the magnetic field produces on the energy of a generic state is a combination of the gyromagnetic interaction and the influence of the space-time geometry. The states which are more affected are those with a maximum value of $J_{R}$. These are the states on the first Regge trajectory, viz. states of the form $\left(b_{1+}^{\dagger}\right)^{k} \tilde{P}|0 ; l=0\rangle$, where $\tilde{P}$ is a product of left-moving creation operators. Such states have $N=J_{R}=k, \tilde{N}=k-m w$. As one gradually increases the magnetic field, the energy decreases until a critical point at which it vanishes. For $k>>m, w$ this occurs when (see eq. (3.42))

$$
\alpha^{\prime} Q f \cong \frac{1}{k-\frac{1}{2}}\left[\frac{1}{2}\left(w r-\frac{m}{r}\right)^{2}+2(k-1)\right] .
$$

Let us consider, for example, the case $r=1$ and $m=w$. Then we have $\alpha^{\prime} Q f \cong 2(k-$ $1) /\left(k-\frac{1}{2}\right)$. As the magnetic field is increased from $2 /\left(\alpha^{\prime} Q\right)-\epsilon$ to $2 /\left(\alpha^{\prime} Q\right)$ an infinite number of zero-energy excitations appear progressively. Similar effect happens for generic values of $r$. On the other hand, instabilities will be expected when the energy will develop an imaginary part, that is, when $\left[E+\kappa^{-1} f\left(\hat{J}_{R}+\alpha^{\prime} f Q\right)\right]^{2}<0$. From eq. (3.42) we see that this can only happen for $\tilde{N}=0$, i.e., when there are no left-moving excitations, and for $f$ greater than the critical value,

$$
f_{\mathrm{cr}}=\frac{Q \hat{J}_{R}-\sqrt{\frac{4}{\alpha^{\prime}}\left(\hat{J}_{R}^{2}-2\right)+2 Q^{2}}}{\hat{J}_{R}^{2}-2}, \quad m w=k>0 .
$$

There will be infinitely many tachyonic instabilities for an arbitrarily small value of the magnetic field. Indeed, in the example with $r=1$ and $m=w$ states, there are an infinite number of tachyons with $\tilde{N}=0, N=m^{2}, J_{R} \cong 2 m /\left(\sqrt{\alpha^{\prime}} f\right)$. The critical magnetic field for a given state will be the lower, the larger the value of its charge.

The fact that in closed string theory a finite magnetic field already produces an infinite number of tachyonic instabilities confirms the picture suggested in ref. [11] on the basis of the linear in $f$ approximation in the simplified model (2.1). This conclusion is different from what happens in the open string case [3],6] where a finite magnetic field can lead only to a finite number of tachyons. We shall further comment on this instability of a constant magnetic field background in closed string theory in Section 6 . 


\section{Partition function on the torus}

Below we shall demonstrate that the results derived in previous section using the lightcone gauge operator approach are consistent with the one-loop (torus) partition function $Z$ calculated in the path integral formalism. We will reproduce the standard relation (see e.g. [38, 39]) between the path integral and operator approach expressions for $Z$ and check its modular invariance and duality invariance in the compact target space direction.2 2

\subsection{Path integral derivation}

We shall take the metric of the torus in the form

$$
\begin{gathered}
d s^{2}=g_{\alpha \beta} d \sigma^{\alpha} d \sigma^{\beta}, \quad 0<\sigma_{\alpha} \leq 1, \quad \tau=\tau_{1}+i \tau_{2}, \\
g_{\alpha \beta}=\left(\begin{array}{cc}
1 & \tau_{1} \\
\tau_{1} & |\tau|^{2}
\end{array}\right), \quad \sqrt{g} g^{\alpha \beta}=\tau_{2}^{-1}\left(\begin{array}{cc}
|\tau|^{2} & -\tau_{1} \\
-\tau_{1} & 1
\end{array}\right) .
\end{gathered}
$$

All the fields are periodic in $\sigma_{\alpha}$ with period 1.23

The Euclidean action and the torus partition function for our model (2.14) are given by

$$
\begin{gathered}
I=\frac{1}{4 \pi \alpha^{\prime}} \int d^{2} \sigma \sqrt{g}\left[\partial_{\alpha} u \partial^{\alpha} v+\partial_{\alpha} x^{i} \partial^{\alpha} x_{i}+f\left(g^{\alpha \beta}+i \frac{\epsilon^{\alpha \beta}}{\sqrt{g}}\right) \epsilon_{i j} x^{i} \partial_{\alpha} x^{j} \partial_{\beta} u\right], \\
Z=\int\left[d^{2} \tau\right] \tilde{Z}(\tau), \quad \tilde{Z}=\int[d u][d v]\left[d x^{i}\right] \exp (-I) .
\end{gathered}
$$

Integration over $v$ produces a $\delta$-function that constrains $u$ to be a zero-mode of the Laplacian on the torus. If $\phi=\frac{1}{2}(u+v)$ is periodic with period $2 \pi R$ it should satisfy the conditions $\phi\left(\sigma_{1}+1, \sigma_{2}\right)=\phi\left(\sigma_{1}, \sigma_{2}\right)+2 \pi R w, \phi\left(\sigma_{1}, \sigma_{2}+1\right)=\phi\left(\sigma_{1}, \sigma_{2}\right)+2 \pi R w^{\prime}$, where $w, w^{\prime}$ are two integer winding numbers. Then the zero modes of $u$ and $v$ on the torus are given by

$$
u_{*}=u_{0}+2 \pi R\left(w \sigma_{1}+w^{\prime} \sigma_{2}\right), \quad v_{*}=v_{0}+2 \pi R\left(w \sigma_{1}+w^{\prime} \sigma_{2}\right) .
$$

After the integration over $v$ and $u$ the action in (4.3) is replaced by $I=I_{*}+I^{\prime}$, where

$$
I_{*}=\frac{1}{4 \pi \alpha^{\prime}} \int d^{2} \sigma \sqrt{g} \partial_{a} u_{*} \partial^{a} v_{*}=\pi r^{2} \tau_{2}^{-1}\left(w^{\prime}-\tau w\right)\left(w^{\prime}-\bar{\tau} w\right), \quad r \equiv R / \sqrt{\alpha^{\prime}},
$$

22 Both symmetries must of course be present in the partition function since our model is based on a $2 d$ diffeomorphism invariant and duality invariant world-sheet action.

23 The correspondence with the Euclidean world sheet notation used in Section 2 is established by setting $\tau=i$ and interchanging $\sigma_{1}$ with $\sigma_{2}$, cf. footnote 2 . 


$$
I^{\prime}=\frac{1}{4 \pi \alpha^{\prime}} \int d^{2} \sigma \tau_{2}^{-1}\left[\left(\partial_{2}-\tau \partial_{1}\right) x^{i}\left(\partial_{2}-\bar{\tau} \partial_{1}\right) x_{i}+f \epsilon_{i j} x^{i}\left(\partial_{2}-\bar{\tau} \partial_{1}\right) x^{j}\left(\partial_{2}-\tau \partial_{1}\right) u_{*}\right] .
$$

The integration over the two coordinates $x^{i}$ gives the following contribution to $\tilde{Z}$ in (4.3)

$$
\begin{gathered}
\tilde{Z}_{x}=\left[\operatorname{det}^{\prime}\left(\Delta_{0}+\mathcal{D}\right) \operatorname{det}^{\prime}\left(\Delta_{0}-\mathcal{D}\right)\right]^{-1 / 2}, \quad \Delta_{0} \equiv-\left(\partial_{2}-\bar{\tau} \partial_{1}\right)\left(\partial_{2}-\tau \partial_{1}\right) \\
\mathcal{D} \equiv i f\left(\partial_{2}-\tau \partial_{1}\right) u_{*}\left(\partial_{2}-\bar{\tau} \partial_{1}\right)=2 \pi i h\left(w^{\prime}-\tau w\right)\left(\partial_{2}-\bar{\tau} \partial_{1}\right), \quad h \equiv f R=\sqrt{\alpha^{\prime}} f r .
\end{gathered}
$$

Expanding $x^{i}$ as

$$
x^{i}=x_{0}^{i}+\sum_{\left(n, n^{\prime}\right) \neq(0,0)} a_{n n^{\prime}}^{i} \exp 2 \pi i\left(n \sigma_{1}+n^{\prime} \sigma_{2}\right)
$$

we get

$$
\operatorname{det}^{\prime}\left(\Delta_{0}+\mathcal{D}\right)=\operatorname{det}^{\prime}\left(\Delta_{0}-\mathcal{D}\right)=\prod_{\left(n, n^{\prime}\right) \neq(0,0)} \frac{\pi^{2}}{\tau_{2}^{2}}\left(n^{\prime}-\bar{\tau} n\right)\left[n^{\prime}-\tau n+h\left(w^{\prime}-\tau w\right)\right]
$$

As a result, we can represent (4.7) as the standard contribution of the two scalars times the "correction" factor $Y^{-1}$

$$
\begin{gathered}
\tilde{Z}_{x}=\left[\operatorname{det}^{\prime} \Delta_{0}\right]^{-1} Y^{-1} \\
\operatorname{det}^{\prime} \Delta_{0}=\tau_{2}^{2} \eta^{2} \bar{\eta}^{2}, \quad \eta(q) \equiv q^{1 / 24} \prod_{n=1}^{\infty}\left(1-q^{n}\right) \equiv q^{1 / 24} f_{0}(q), \quad q=e^{2 \pi i \tau}, \\
Y \equiv \prod_{\left(n, n^{\prime}\right) \neq(0,0)}\left[1+h \frac{w^{\prime}-\tau w}{n^{\prime}-\tau n}\right] .
\end{gathered}
$$

The non-trivial part of the $\tau$-integrand in (4.3) which contains the dependence on $R$ and $f$ is given by the sum over windings of the product of the semiclassical factor (exponential of the classical zero mode a ction $I_{*}$ in (4.5) and $Y^{-1}$ (we include also the standard zero-mode f actor $r$ )

$$
\begin{aligned}
W(r, h ; \tau, \bar{\tau})= & r \sum_{w, w^{\prime}=-\infty}^{\infty} \exp \left[-\pi r^{2} \tau_{2}^{-1}\left(w^{\prime}-\tau w\right)\left(w^{\prime}-\bar{\tau} w\right)\right] \\
& \times \prod_{\left(n, n^{\prime}\right) \neq(0,0)}\left[1+h \frac{w^{\prime}-\tau w}{n^{\prime}-\tau n}\right]^{-1} .
\end{aligned}
$$

This representation makes it explicit that the partition function is modular invariant for arbitrary $r$ and $h$ (as in Section 3 we add 22 extra free scalar degrees of freedom to satisfy the zero central charge condition),

$$
Z=c_{1} \int d^{2} \tau \tau_{2}^{-14} e^{4 \pi \tau_{2}}\left|f_{0}\left(e^{2 \pi i \tau}\right)\right|^{-48} W(r, h ; \tau, \bar{\tau}) .
$$


In fact, the transformations $\tau \rightarrow \tau+1$ and $\tau \rightarrow-1 / \tau$ are "undone" by the redefinitions of the summation parameters: $w \rightarrow-w^{\prime}, w^{\prime} \rightarrow w, n \rightarrow-n^{\prime}, n^{\prime} \rightarrow n$.

To find the explicit representation for $Y$ one should be careful to use a reparametrisation invariant (modular invariant) regularisation of the infinite product in (4.10). Let us first note that

$$
\begin{aligned}
& U(\tau, \bar{\tau}, \chi) \equiv \prod_{\left(n, n^{\prime}\right) \neq(0,0)}\left(n^{\prime}-\bar{\tau} n\right)\left(n^{\prime}-\tau n+\chi\right) \\
& =\prod_{k \neq 0} k(k+\chi) \prod_{n \neq 0, n^{\prime}}\left(n^{\prime}-\bar{\tau} n\right)\left(n^{\prime}-\tau n+\chi\right) .
\end{aligned}
$$

Summing first over $k$ and $n^{\prime}$ using that

$$
\prod_{n=-\infty}^{\infty}(n+\chi)=\chi \prod_{n=1}^{\infty}\left(-n^{2}\right)\left(1-\frac{\chi^{2}}{n^{2}}\right)=2 i \sin \pi \chi
$$

defining the product $\prod_{n \neq 0} \exp (i \pi \bar{\tau} n) \exp (-i \pi \tau n+i \pi \chi)$ as $\exp \left[2 \pi \tau_{2} \sum_{n \neq 0}\left(n+i \chi / 2 \tau_{2}\right)\right]$, and using the generalised $\zeta$-function regularisation as in sect. 3.3 we finally obtain

$$
\begin{aligned}
Y \equiv \frac{U(\tau, \bar{\tau}, \chi)}{U(\tau, \bar{\tau}, 0)}= & e^{\frac{\pi \chi^{2}}{2 \tau_{2}}} \frac{\sin \pi \chi}{\pi \chi} \prod_{n=1}^{\infty} \frac{\left(1-\rho^{-1} q^{n}\right)\left(1-\rho q^{n}\right)}{\left(1-q^{n}\right)^{2}} \\
& =e^{\frac{\pi \chi^{2}}{2 \tau_{2}}} \frac{\theta_{1}(\chi \mid \tau)}{\chi \theta_{1}^{\prime}(0 \mid \tau)},
\end{aligned}
$$

where

$$
\tilde{w} \equiv w^{\prime}-\tau w, \quad \rho \equiv \exp (2 \pi i h \tilde{w}), \quad q=\exp (2 \pi i \tau), \chi \equiv h \tilde{w} .
$$

The resulting expression for $Z$ (4.15) is manifestly modular invariant and will be shown below to be in agreement with the operator formalism.

\subsection{Equivalence with operator formalism result}

In the operator formalism, the one-loop partition function is obtained by using the Hamiltonian to propagate the states along the cylinder and identifying its ends

$$
Z=\int \frac{d^{2} \tau}{\tau_{2}} \int d E \prod_{a=1}^{22} d p_{a} \sum_{m, w=-\infty}^{\infty} \operatorname{Tr}\left(q^{\hat{L}_{0}} \bar{q}^{\hat{\tilde{L}}_{0}}\right)
$$

where $q=\exp (2 \pi i \tau)$ and $\hat{L}_{0}$ and $\hat{\tilde{L}}_{0}$ are the Virasoro operators constructed in Section 3 $($ see $(3.33),(3.34))$

$$
\hat{L}_{0}=\frac{1}{4} \alpha^{\prime}\left[-\left(E+f \hat{J}_{R}\right)^{2}+p_{a}^{2}\right]-1-\frac{1}{8} \gamma^{2}+\mathcal{L}_{0}
$$




$$
\begin{gathered}
\hat{\tilde{L}}_{0}=\frac{1}{4} \alpha^{\prime}\left[-\left(E+f \hat{J}_{R}\right)^{2}+p_{a}^{2}\right]-1-\frac{1}{8} \gamma^{2}+\tilde{\mathcal{L}}_{0} \\
\mathcal{L}_{0}=\frac{1}{4}\left(m r^{-1}+w r-\sqrt{\alpha^{\prime}} f \hat{J}_{R}\right)^{2}-m w+N, \quad \tilde{\mathcal{L}}_{0}=\frac{1}{4}\left(m r^{-1}+w r-\sqrt{\alpha^{\prime}} f \hat{J}_{R}\right)^{2}+\tilde{N} .
\end{gathered}
$$

It is convenient to express the exponential factor containing $\gamma^{2}$ in the following way:

$$
\exp \left(\frac{1}{2} \pi \tau_{2} \gamma^{2}\right)=\sqrt{\tau_{2}} \int d x \exp \left(-\frac{1}{2} \pi \tau_{2} x^{2}-\pi \tau_{2} \gamma x\right) .
$$

The term $\pi \tau_{2} \gamma x$ can be absorbed into a redefinition of $\hat{J}_{R} \rightarrow \hat{J}_{R}^{\prime} \equiv \hat{J}_{R}-\frac{1}{2} x$, as can be easily verified. Integrating over $E, p_{a}$ we get (4.19) with an extra measure factor $\tau_{2}^{-23 / 2}$ and the same trace over the oscillator states with $\hat{L}_{0}, \hat{\tilde{L}}_{0}$ replaced by $\mathcal{L}_{0}, \tilde{\mathcal{L}}_{0}$. The next step is to perform the Poisson resummation (or duality transformation on the world sheet), trading the sum over the discrete loop momentum $m$ for the sum over the conjugate winding number $w^{\prime}$ :

$$
\begin{gathered}
\sum_{m=-\infty}^{\infty} F(m)=\sum_{w^{\prime}=-\infty}^{\infty} \int d s F(s) \exp \left(2 \pi i s w^{\prime}\right), \\
F(m)=\exp \left[\frac{1}{2} \pi i(\tau-\bar{\tau})\left(m r^{-1}+w r-\sqrt{\alpha^{\prime}} f \hat{J}_{R}^{\prime}\right)^{2}-2 \pi i m w \tau\right] .
\end{gathered}
$$

Integrating over $s$ we get (up to a numerical factor)

$$
\frac{r}{\sqrt{\tau_{2}}} \exp \left[-\frac{\pi r^{2}}{\tau_{2}}\left(w^{\prime}-\tau w\right)\left(w^{\prime}-\bar{\tau} w\right)\right] \operatorname{Tr}\left\{q^{N} \bar{q}^{\tilde{N}} \exp \left[2 \pi i\left(w^{\prime}-\tau w\right) r \sqrt{\alpha^{\prime}} f \hat{J}_{R}^{\prime}\right]\right\} .
$$

The first exponential factor is just the standard semiclassical contribution in (4.14). The trace produces the non-trivial magnetic field dependent contribution (see (3.37), (3.43))

$$
\begin{aligned}
& X(q, \bar{q}, \tilde{w} h) \equiv \sum_{l=0}^{\infty} \operatorname{Tr}\left\{q^{N} \bar{q}^{\tilde{N}} \exp \left[2 \pi i \tilde{w} h\left(-l-\frac{1}{2}-\frac{1}{2} x+S_{R}\right)\right]\right\} \\
& =X(q, \bar{q}, 0) e^{-\pi i h \tilde{w} x} \frac{\pi h \tilde{w}}{\sin (\pi h \tilde{w})} \prod_{n=1}^{\infty} \frac{\left(1-q^{n}\right)^{2}}{\left(1-\rho q^{n}\right)\left(1-\rho^{-1} q^{n}\right)}
\end{aligned}
$$

which, after integrating over $x$, is equal to $Y^{-1}$ (see (4.17)). The non-trivial zero-mode factor of $h \tilde{w}$ (necessary for the regular $f \rightarrow 0$ limit) originates from the correct normalisation of the trace consistent with the zero-field limit: note that in (2.40) or (3.28) one made a rescaling of the original $x$-field modes by the factor of $f p_{v}$ or $\frac{1}{2} f p_{+}$or, in the present setting, $f\left(\partial_{2}-\tau \partial_{1}\right) u_{*}=f \tilde{w}$ (see (4.4)). The zero mode normalisation condition also makes the trace in (4.24) implicitly $\tau_{2}$-dependent, leading to an extra $1 / \tau_{2}$ factor in $X(q, \bar{q}, 0)$ (see e.g. [40] for a discussion of related normalisation issues). 
We conclude that the path integral and operator approaches give indeed equivalent expressions for the one-loop partition function depending on radius $R=\sqrt{\alpha^{\prime}} r$ of the compact dimension and the magnetic field $f$ (see (4.15), (4.13))

$$
\begin{gathered}
Z(R, f)=c_{1} \int d^{2} \tau \tau_{2}^{-14} e^{4 \pi \tau_{2}}\left|f_{0}\left(e^{2 \pi i \tau}\right)\right|^{-48} W(R, f ; \tau, \bar{\tau}), \\
W=\frac{R}{\sqrt{\alpha^{\prime}}} \sum_{w, w^{\prime}=-\infty}^{\infty} \exp \left[-\frac{\pi R^{2}}{\alpha^{\prime} \tau_{2}}\left(w^{\prime}-\tau w\right)\left[\left(w^{\prime}-\bar{\tau} w\right)+\frac{1}{2} \alpha^{\prime} f^{2}\left(w^{\prime}-\tau w\right)\right]\right] \\
\times \frac{f R\left(w^{\prime}-\tau w\right) \theta_{1}^{\prime}(0 \mid \tau)}{\theta_{1}\left[f R\left(w^{\prime}-\tau w\right) \mid \tau\right]} .
\end{gathered}
$$

The path integral derivation makes explicit the modular invariance of $Z$ (see (4.14)), while the operator derivation demonstrates its duality invariance ( $Z$ in (4.19) is manifestly invariant under $r \rightarrow 1 / r, m \rightarrow w$ ). In the limit $f=0$ (4.25) reduces to the standard partition function of the bosonic string with one compact dimension.

In the limit of the non-compact $\phi$-dimension, $R \rightarrow \infty$, the dependence on $f$ disappears and the partition function becomes equivalent to the flat space one.24 In fact, the duality invariance of $Z$ implies that $R \rightarrow \infty$ limit is equivalent to $R \rightarrow 0$. In the latter case $h \rightarrow 0$ so that $Y \rightarrow 1$ (see (4.13)) and the final expression for $Z$ is the usual flat space one, i.e. a constant times $R^{-1} \rightarrow \infty$ (diverging as a volume).

The partition function (4.25) (see also (4.26) below) has "extra" poles at rational values of $h=f R$. These singularities originate (upon integrating over $E$ ) from contributions of the extra zero modes (associated with a translational invariance in the $x, x^{*}$-plane, see Section 3.1) that appear in the string solution (cf. (3.11)) in a sector with $p_{+} f=2 n$, i.e. $\alpha^{\prime} f(E+Q)=2 n$ ( $n=$ integer). A possible physical role of these special $f R$ points (or special values of momentum $p_{+}$of a free string) deserves further clarification.

\subsection{Tachyonic instabilities}

The magnetic instability of our background (implied by the presence of tachyons in the spectrum found in Section 3) is reflected in extra singularities (or imaginary parts) in $Z$. This can be seen explicitly from the behavior of the integrand for large $\tau_{2}$ if one starts from the operator formalism representation (4.19) and performs the trace before integrating over $E$. One finds

$$
Z=\int \frac{d^{2} \tau}{\tau_{2}^{13}} \int d E \sum_{m, w=-\infty}^{\infty} e^{\pi \tau_{2}\left[4+\alpha^{\prime}\left(E^{2}-Q^{2}\right)+\frac{1}{2} \gamma^{2}\right]-2 \pi i \tau m w}\left|f_{0}\left(e^{2 \pi i \tau}\right)\right|^{-48}
$$

24 This agrees with the observation made in 250 that the torus partion function for the model of [17] is equal to its flat space value. 


$$
\times \frac{\lambda}{\sinh \lambda} \prod_{n=1}^{\infty}\left(1-q^{n}\right)^{2}\left[\left(1-e^{2 \lambda} q^{n}\right)\left(1-e^{-2 \lambda} q^{n}\right)\right]^{-1}
$$

where $\lambda \equiv \pi \tau_{2} \alpha^{\prime}(Q+E) f=\pi \tau_{2} \gamma, \quad Q \equiv(m / r+w r) / \sqrt{\alpha^{\prime}}$. Expanding the different factors in powers of $q$ and $\bar{q}$, one gets a sum in which each term represents the contribution of a corresponding state in the spectrum.

In Section 3 we have seen that the energy of a state is more affected by the magnetic field (relative to the $f=0$ energy) the higher is the corresponding value of $J_{R}$. In eq. (4.27) the states with highest angular momentum for a given level correspond to the terms obtained from the leading terms in all the factors except $\left(1-e^{-2 \lambda} q\right)^{-1}$. They correspond to the $\tilde{N}=0$ states discussed in Section 3, i.e. states on the first Regge trajectory. We can represent their contribution to $Z$ as follows

$$
Z=\sum_{m, w=-\infty}^{\infty} \int d E \int d \tau_{1} e^{2 \pi i \tau_{1}(k-m w)} \int \frac{d \tau_{2}}{\tau_{2}^{13}} \sum_{k=0}^{\infty} e^{-\pi \tau_{2} M_{k}^{2}}+\ldots
$$

with

$$
M_{k}^{2}=-4-\alpha^{\prime} E^{2}+\frac{m^{2}}{r^{2}}+w^{2} r^{2}-2 \alpha^{\prime}(Q+E) f\left(k-\frac{1}{2}\right)+2 k-\frac{1}{2} \gamma^{2} .
$$

Integrating over $\tau_{1}$ and $E$ we obtain

$$
\begin{gathered}
Z \sim \kappa^{-1 / 2} \sum_{m, w=-\infty}^{\infty} \int \frac{d \tau_{2}}{\tau_{2}^{27 / 2}} \sum_{k=0}^{\infty} e^{-\pi \tau_{2} M_{k}^{\prime 2}} \delta_{k, m w}+\ldots, \\
M_{k}^{\prime 2}=-4+\kappa^{-1}\left[\frac{m}{r}+w r-\sqrt{\alpha^{\prime}} f\left(k-\frac{1}{2}\right)\right]^{2}, \quad \kappa=1+\frac{1}{2} \alpha^{\prime} f^{2} .
\end{gathered}
$$

The partition function has new divergences when $M_{k}^{\prime 2}<0$. As expected, this occurs exactly at the critical magnetic field (3.49) when the energy develops an imaginary part.

\section{Heterotic string generalisations}

Let us now follow Section 2.1 and address the question of how to embed a constant magnetic field background in a closed superstring or heterotic string theory. Since there are no fundamental gauge fields in the type II superstring theory the only option is to couple the gauge field to a compact Kaluza-Klein coordinate as in the bosonic case. The corresponding world sheet theory is then the direct $(1,1)$ supersymmetric extension of the bosonic $\sigma$-model (2.5).

As for the heterotic string theory, here there are more options. One may consider the $(1,0)$ and $(0,1)$ supersymmetric extensions of the bosonic model (and to add interactions in the internal fermionic sector if necessary for conformal invariance) thus getting the magnetic field again from the Kaluza-Klein sector (Section 5.1). Another possibility is 
to embed the magnetic field directly into the heterotic gauge group, i.e. to couple $A_{i}$ to internal fermions. This second approach is more appealing from a "phenomenological" viewpoint since in this case the corresponding $\sigma$-model background will describe the effect on the geometry when the usual (i.e. coming from $U(1)_{\text {e.m. }}$ ) magnetic field is turned on (cf. ref. [10]).

If one starts with the flat-space heterotic string action [9] and introduces the magnetic field in the internal fermionic sector

$$
\begin{gathered}
I=\frac{1}{\pi \alpha^{\prime}} \int d^{2} \sigma\left[-\partial t \bar{\partial} t+\partial x_{i} \bar{\partial} x^{i}-\lambda_{L}^{t} \partial \lambda_{L}^{t}+\lambda_{L i} \partial \lambda_{L}^{i}\right. \\
\left.+\psi_{R I}\left(\delta^{I J} \bar{\partial}+V_{i}^{I J} \bar{\partial} x^{i}\right) \psi_{R J}-\frac{1}{2} \mathcal{F}_{i j}^{I J}(V) \psi_{R I} \psi_{R J} \lambda_{L}^{i} \lambda_{L}^{j}\right],
\end{gathered}
$$

where $V_{i}^{I J}=T^{I J} A_{i}$ ( $T$ is proportional to a $U(1)$ generator of the gauge group), then, as in the bosonic case (2.1), such model will not be conformally invariant since the magnetic field will deform the space-time geometry.

To find a conformally invariant modification of (5.1) one may consider $\psi_{R}$ as playing the role of the internal Kaluza-Klein field $\phi$ of the bosonic model (2.4),(2.18). 2.2 Assuming that the $\phi$-terms of the second line in (2.18) are "fermionised" and supersymmetrising (in the $(0,1)$ way $[42,43])$ the $t, x^{i}$-terms of the first line in (2.18) we obtain the following heterotic $\sigma$-model action

$$
\begin{gathered}
I^{\prime}=\frac{1}{\pi \alpha^{\prime}} \int d^{2} \sigma\left[-\left(\partial t+A_{i} \partial x^{i}\right)\left(\bar{\partial} t+A_{i} \bar{\partial} x^{i}\right)+\partial x^{i} \bar{\partial} x_{i}+A_{i}\left(\partial x^{i} \bar{\partial} t-\bar{\partial} x^{i} \partial t\right)\right. \\
\left.-\lambda_{L}^{\hat{t}} \partial \lambda_{L}^{\hat{t}}+\lambda_{L}^{i}\left[\delta_{i j} \partial+F_{i j}\left(\partial t+\frac{1}{2} A_{k} \partial x^{k}\right)\right] \lambda_{L}^{j}+\bar{\psi}_{R}\left[\bar{\partial}-i e_{0}\left(A_{i} \bar{\partial} x^{i}-\frac{1}{2} F_{i j} \lambda_{L}^{i} \lambda_{L}^{j}\right)\right] \psi_{R}\right],
\end{gathered}
$$

where $\lambda_{L}^{\hat{t}} \equiv \lambda_{L}^{t}+A_{i} \lambda_{L}^{i}$. We have assumed that $T^{I J}=e_{0} \epsilon^{I J}, I, J=1,2$, and combined the two Majorana-Weyl spinors $\psi_{R I}$ into a single Weyl one, $\psi_{R}=\left(\psi_{R 1}+i \psi_{R 2}\right) / \sqrt{2}$ (we did not write down explicitly the decoupled free fermionic terms). The geometrical background corresponding to this model is thus the same as of the dimensionally reduced bosonic theory (2.19). The gauge invariance $A_{i}^{\prime}=A_{i}+\partial_{i} \alpha, t^{\prime}=t-\alpha, \alpha=\alpha(x)$ (cf. (2.6)) is now maintained due to the cancellation of the classical bosonic anomaly (in the antisymmetric tensor sector) against the one-loop chiral fermionic $\left(\psi_{R}\right)$ anomaly.

Similarly, the conformal anomaly which was previously absent because of the contributions of $\phi$ is now cancelled by the contributions of $\psi_{R}$ (coming from one loop order higher).2 In fact, the leading-order condition of conformal invariance of this model

25 A different conformal embedding of a monopole-type magnetic field into the gauge sector of the heterotic string theory which uses $S U(2)$ WZW model was recently discussed in [41.

26 This cancellation between different bosonic and fermionic loop orders is a direct consequence of the use of bosonisation and takes place also for analogous heterotic string solutions of [44, 45]. 
$R_{\mu \nu}-\frac{1}{4} \hat{H}_{\mu \lambda \rho} \hat{H}_{\nu}^{\lambda \rho}-\frac{1}{4} \alpha^{\prime} \mathcal{F}(V)_{\mu \lambda}^{I J} \mathcal{F}(V)_{I J \nu}{ }^{\lambda}+\ldots=0$ is satisfied provided $e_{0}^{2}=2 / \alpha^{\prime}$. This is identical to the bosonic model condition (2.27) with the gauge field term now coming from the two-loop fermionic contribution (the leading terms in the effective actions of the dimensionally reduced bosonic model and a heterotic model with an abelian vector field are formally the same if $\left.e_{0}^{2} \sim \alpha^{\prime}\right)$.27 In Section 5.2 we shall give a systematic argument that (5.2) indeed represents a conformal heterotic model.

\subsection{Magnetic field from Kaluza-Klein bosonic sector}

The generalization of (2.5) to the case of the closed superstring theory is straightforward. The $(1,1)$ supersymmetric $\sigma$-model action [46] in the component representation is (here $\hat{\omega}_{ \pm n \mu}^{m}=\omega_{n \mu}^{m} \pm \frac{1}{2} H_{n \mu}^{m}$ is the generalised Lorentz connection, $m, n, \ldots$, are tangent space indices)

$$
\begin{gathered}
I_{(1,1)}=\frac{1}{\pi \alpha^{\prime}} \int d^{2} \sigma\left[\left(G_{\mu \nu}+B_{\mu \nu}\right)(x) \partial x^{\mu} \bar{\partial} x^{\nu}+\lambda_{R m}\left[\delta_{n}^{m} \bar{\partial}+\hat{\omega}_{-n \mu}^{m}(x) \bar{\partial} x^{\mu}\right] \lambda_{R}^{n}\right. \\
\left.+\lambda_{L m}\left[\delta_{n}^{m} \partial+\hat{\omega}_{+n \mu}^{m}(x) \partial x^{\mu}\right] \lambda_{L}^{n}-\frac{1}{2} \hat{R}_{+m n p q} \lambda_{L}^{m} \lambda_{L}^{n} \lambda_{R}^{p} \lambda_{R}^{q}\right] .
\end{gathered}
$$

In the case of the model (2.5) we get explicitly 28

$$
\begin{gathered}
I_{(1,1)}=\frac{1}{\pi \alpha^{\prime}} \int d^{2} \sigma\left(\partial u \bar{\partial} v+2 A_{i} \partial u \bar{\partial} x^{i}+\partial x^{i} \bar{\partial} x_{i}+\lambda_{R}^{u} \bar{\partial} \lambda_{R}^{\hat{v}}+\lambda_{R i} \bar{\partial} \lambda_{R}^{i}\right. \\
\left.+F_{i j} \bar{\partial} x^{j} \lambda_{R}^{u} \lambda_{R}^{i}+\lambda_{L}^{\hat{v}} \partial \lambda_{L}^{u}+\lambda_{L i} \partial \lambda_{L}^{i}-F_{i j} \partial u \lambda_{L}^{i} \lambda_{L}^{j}-\frac{1}{2} \partial_{i} F_{j k} \lambda_{L}^{j} \lambda_{L}^{k} \lambda_{R}^{u} \lambda_{R}^{i}\right) .
\end{gathered}
$$

Like the bosonic model (2.5), this model is conformal to all loop orders provided $\partial_{i} F^{i j}=0$ [12] (as can be shown, e.g., by repeating the bosonic argument in terms of $u, v, x^{i}$ replaced by superfields). The quartic fermionic term in (5.4) is absent in the case of the constant magnetic field $F_{i j}=$ const. A simple test that this model is conformal follows from the path integral: if one ignores the sources for $v, \lambda_{R}^{\hat{v}}$ the integrals over $v$ and $\lambda_{R}^{\hat{v}}$ effectively

27 A simple indication that such heterotic model is, like the bosonic one, conformally invariant is that the contributions of one-loop diagrams with an internal $t$-propagator can be cancelled against the contributions of two-loop diagrams with the $t$-propagator replaced by the $\psi_{R}$-loop (playing here the role of the $\phi$-propagator of the bosonic model).

28 The non-vanishing components of the connection and curvature are: $\hat{\omega}_{-\hat{u} \hat{i}}=F_{i j} d x^{j}, \hat{\omega}_{+\hat{i} \hat{j}}=$ $-F_{i j} d u, \quad \hat{R}_{+\hat{j} \hat{k} \hat{u} \hat{i}}=\partial_{i} F_{j k}$. Hats here indicate the tangent space indices corresponding to the vierbein $e^{\hat{u}}=d u, e^{\hat{v}}=d v+2 A_{i} d x^{i}, e^{\hat{i}}=d x^{i}$. The only fermionic vierbein component $\left(\lambda^{m} \equiv\right.$ $e_{\mu}^{m} \lambda^{\mu}$ ) which is different from the one with a coordinate index is thus $\lambda^{\hat{v}}=\lambda^{v}+2 A_{i} \lambda^{i}$ (we shall consider $\lambda^{\hat{v}}$ as a new field replacing $\lambda^{v}$ ). 
"freeze" out $u$ and $\lambda_{R}^{u}$ and thus the interaction terms $F_{i j} \bar{\partial} x^{j} \lambda_{R}^{u} \lambda_{R}^{i}$ and $F_{i j} \partial u \lambda_{L}^{i} \lambda_{L}^{j}$ in (5.3) do not produce non-trivial contributions.

Eq. (5.4) can be also interpreted as the action of a heterotic $\sigma$-model [42,43] corresponding to a "symmetric" heterotic solution obtained by the standard embedding of a closed superstring solution into the heterotic string theory $\left(\lambda_{L}^{\mu}\right.$ then play the role of the internal fermions and $V_{u}^{i j}=\hat{\omega}_{+u}^{i j}=-F^{i j}$ - of the internal gauge field). This solution preserves extended space-time supersymmetry [16].

In addition, there are two non-trivial "asymmetric" heterotic models corresponding to $(1,0)$ and $(0,1)$ supersymmetric truncations of (5.4) [12]. Both represent exact heterotic string solutions when combined with a free internal fermionic sector (i.e. there is no need to introduce non-trivial internal gauge field background).29 For example, the action for the $(1,0)$ heterotic model reads

$$
\begin{gathered}
I_{(1,0)}=\frac{1}{\pi \alpha^{\prime}} \int d^{2} \sigma\left[\partial u \bar{\partial} v+2 A_{i} \partial u \bar{\partial} x^{i}+\partial x^{i} \bar{\partial} x_{i}\right. \\
\left.+\lambda_{R}^{u} \bar{\partial} \lambda_{R}^{\hat{v}}+\lambda_{R i} \bar{\partial} \lambda_{R}^{i}+F_{i j} \bar{\partial} x^{j} \lambda_{R}^{u} \lambda_{R}^{i}+\psi_{L I} \partial \psi_{L}^{I}\right],
\end{gathered}
$$

where $\psi_{L}^{I}$ are the fermions of the internal sector.

\subsection{Magnetic field from the internal fermionic sector}

In the above heterotic solutions the magnetic field came from the Kaluza-Klein sector. Let us now return to the discussion at the beginning of this section and argue that the model (5.2) in which the magnetic field is embedded into the internal gauge sector also represents an exact heterotic string solution. Let us start with the $(0,1)$ supersymmetric truncation of (5.4) which, as explained above, is $\left(\right.$ for $\left.\partial_{i} F^{i j}=0\right)$ an exact conformal model

$$
I_{(0,1)}=\frac{1}{\pi \alpha^{\prime}} \int d^{2} \sigma\left(\partial u \bar{\partial} v+2 A_{i} \partial u \partial x^{i}+\partial x^{i} \bar{\partial} x_{i}+\lambda_{L}^{\hat{v}} \partial \lambda_{L}^{u}+\lambda_{L i} \partial \lambda_{L}^{i}-F_{i j} \partial u \lambda_{L}^{i} \lambda_{L}^{j}\right)
$$

Changing the variables to $\phi, t, \lambda_{L}^{\hat{\phi}}, \lambda_{L}^{\hat{t}} \quad\left(v, u=\phi \pm t, \lambda_{L}^{\hat{v}, u}=\lambda_{L}^{\hat{\phi}} \pm \lambda_{L}^{\hat{t}}, \lambda_{L}^{\hat{t}, \hat{\phi}}=\lambda_{L}^{t, \phi}+A_{i} \lambda_{L}^{i}\right.$, cf. (2.4) and separating the $\phi$-dependent terms we get

$$
\begin{gathered}
I_{(0,1)}=\frac{1}{\pi \alpha^{\prime}} \int d^{2} \sigma[ \\
-\partial t \bar{\partial} t-2 A_{i} \partial t \bar{\partial} x^{i}+\partial x^{i} \bar{\partial} x_{i}-\lambda_{L}^{\hat{t}} \partial \lambda_{L}^{\hat{t}}+\lambda_{L i} \partial \lambda_{L}^{i}+F_{i j} \partial t \lambda_{L}^{i} \lambda_{L}^{j} \\
\left.+\lambda_{L}^{\hat{\phi}} \partial \lambda_{L}^{\hat{\phi}}+\partial \phi \bar{\partial} \phi+2\left(A_{i} \bar{\partial} x^{i}-\frac{1}{2} F_{i j} \lambda_{L}^{i} \lambda_{L}^{j}\right) \partial \phi\right] .
\end{gathered}
$$

29 The $(1,0)$ truncation also formally preserves space-time supersymmetry and has extended world-sheet supersymmetry. This is not surprising given that our model with $F_{i j}=$ const is equivalent (at least in the non-compact case) to a non-semisimple WZW model (see Section 2.2). 
The final key step is to observe that since $\lambda_{L}^{\hat{\phi}}$ is completely decoupled, we can now try to fermionise the compact coordinate $\phi$ without breaking $(0,1)$ world sheet supersymmetry (it will act only on the rest of the fields excluding $\lambda_{L}^{\hat{\phi}}$ and the fermionic counterpart of $\phi$ ) and conformal invariance. The scheme in which the resulting model will be conformally invariant will depend on a choice of a scheme used in the fermionisation process.

The action for a periodic real boson $\varphi(0<\varphi \leq 2 \pi)$

$$
I_{B}=\frac{1}{2 \pi} \int d^{2} \sigma(\partial \varphi \bar{\partial} \varphi+2 \bar{B} \partial \varphi+B \bar{B})=\frac{1}{2 \pi} \int d^{2} \sigma[(\partial \varphi+B)(\bar{\partial} \varphi+\bar{B})-F(B) \varphi]
$$

where $B, \bar{B}$ are the components of an external $2 \mathrm{~d}$ gauge field $(F(B) \equiv \partial \bar{B}-\bar{\partial} B)$, is equivalent (in the sense of equality of the corresponding generating functionals, and, in particular, the partition functions) to the action for the two Weyl fermions (see e.g. [47] and refs. there)

$$
I_{F}=\frac{1}{2 \pi} \int d^{2} \sigma\left[\bar{\psi}_{R}(\bar{\partial}-i \bar{B}) \psi_{R}+\bar{\psi}_{L} \partial \psi_{L}\right] .
$$

We are assuming that the chiral fermionic determinant is defined in the "left-right decoupled" scheme in which a specific local counterterm is added to the "core" non-local part $\sim \int \bar{B}(\partial / \bar{\partial}) \bar{B}$ Since $\phi$ in (5.7) was taken to be periodic with period $2 \pi R$, it can be fermionised provided $R^{2}=\alpha^{\prime} / 2$ (we do not introduce the Thirring coupling term). Comparing (5.7) with (5.8) $(\phi=R \varphi)$ we learn that $\bar{B}=R^{-1}\left(A_{i} \bar{\partial} x^{i}-\frac{1}{2} F_{i j} \lambda_{L}^{i} \lambda_{L}^{j}\right)$. Since the supersymmetry should be present only in the left sector we should take $B=R^{-1} A_{i} \partial x^{i}$. The resulting conformal action is thus (we drop the free $\psi_{L}, \lambda_{L}^{\hat{\phi}}$-contributions)

$$
\begin{gathered}
I_{(0,1)}^{\prime}=\frac{1}{\pi \alpha^{\prime}} \int d^{2} \sigma\left[-\partial t \bar{\partial} t-2 A_{i} \partial t \bar{\partial} x^{i}+\left(\delta_{i j}-A_{i} A_{j}\right) \partial x^{i} \bar{\partial} x^{j}\right. \\
-\lambda_{L}^{\hat{t}} \partial \lambda_{L}^{\hat{t}}+\lambda_{L i} \partial \lambda_{L}^{i}+F_{i j} \partial t \lambda_{L}^{i} \lambda_{L}^{j}+\frac{1}{2} F_{i j} A_{k} \partial x^{k} \lambda_{L}^{i} \lambda_{L}^{j}
\end{gathered}
$$

30 Such scheme was used also in a similar context in [44. In general, the bosonisation/fermionisation rule is not unique: there is a freedom of adding local counterterms to the non-local part of the chiral fermionic determinant. Their choice depends on symmetry conditions which are assumed, i.e. on definitions of the space-time fields (or couplings of the theory). For example, if one adopts the "minimal" (vector-like) scheme (used, e.g., in [47,45]) in which there is no $B \bar{B}$ term in (5.8) then instead of (5.10) below one ends up with the action where the target space metric does not contain the "Kaluza-Klein" $A_{i} A_{j}$ - term and thus is not invariant under the abelian gauge transformations. The two schemes are related by the field (metric) redefinition, $G_{i j}^{\prime}=G_{i j}+A_{i} A_{j}$. Such redefinition is also related to a restoration of the world-sheet supersymmetry not apparent in this "minimal" scheme [48]. Note that in the present case this redefinition is not suppressed by an extra power of $\alpha^{\prime}$ and thus the validity of the use of the second scheme is not clear. 


$$
\left.+\bar{\psi}_{R}\left(\bar{\partial}-i e_{0} A_{i} \bar{\partial} x^{i}\right) \psi_{R}+\frac{1}{2} i e_{0} F_{i j} \bar{\psi}_{R} \psi_{R} \lambda_{L}^{i} \lambda_{L}^{j}\right], \quad e_{0} \equiv R^{-1}=\sqrt{2 / \alpha^{\prime}} .
$$

This is the same as the $(1,0)$ supersymmetric heterotic action (5.2) which we have suggested above. The complete anomaly-free heterotic model is obtained by assuming that $i=1, . ., 9$ and adding to (5.10) extra free 30 Majorana-Weyl fermions in the right sector.

It is clear that the direct supersymmetrisations of our model (5.4), (5.5), (5.6) can be solved and quantised (e.g. using the superfield formulation) in the same way as this was done in the bosonic case in Sections 3,4. Since the heterotic model (5.2), (5.10) is closely related to (5.6), the same should apply to it as well. The models will be explicitly unitary in the $(1,0)$ superfield version of light-cone gauge. Such direct solution will give an explicit definition of the corresponding CFT (resolving the scheme ambiguity in a particular way).

\section{Conclusions}

The appearance of tachyons beyond some finite values of the magnetic field, and the fact that the partition function develops new divergences precisely at this value, suggest the presence of a phase transition. These are the same indications that lead one to think that there is a new, more symmetric string phase beyond the Hagedorn temperature [49]. The present model is exactly solvable and thus may provide a framework to study explicitly possible emergence of such new symmetries. The basic idea is to use the magnetic field as a probe. This can be done, for example, to reveal the hidden (spontaneously broken) gauge symmetry of the $S U(2) \times U(1)$ GSW model [37].

As we have have seen in Section 3, the incorporation of a non-trivial geometry demanded in the case of constant magnetic field background by the closed string field equations has produced some substantial changes compared to the open string case. The instability is notoriously enhanced: while in the open string theory it takes an infinite magnetic field in order to get an infinite number of tachyonic states, in the closed string theory an arbitrarily small magnetic field already produces an infinite number of tachyonic instabilities. It would be interesting to study possible consequences of this instability in quantised closed string propagation in a magnetic field. Above a critical value of the field, the tachyonic particles should condense, causing a phase transition. In string theory this may probably be described by correlators with multiple insertion of tachyon vertex operators (see also [49]). A close analogue is the phase transition that takes place in type-I superconductors as the magnetic field is increased. In ref. [11] a phase diagram of a closed string gas was obtained and it was argued that the phase transition is of first order with a large latent heat. It would be interesting to investigate these issues within the present exactly solvable model.

There are a number of other open problems which deserve investigation. 
- In Section 5 we have constructed several supersymmetric extensions of the bosonic model. It is important to compute the spectrum and the partition function of these models and to study their properties. In the case of the open superstring string theory in a constant magnetic field [3,6] the spectrum displays the same qualitative features as in the bosonic case, in particular, the emergence of the tachyon instabilities at critical values of the field. It is plausible that the spectra of the heterotic string models discussed in Section 5 will also be analogous to the bosonic model spectrum, i.e., in particular, will exhibit similar instabilities.

- It would be important to clarify the implications of our model for the value of the $g$-factor in closed string theories and for possible "string states - black holes" connection. We have seen that the space-time (2.19) describing response of geometry to a uniform magnetic field is represented by a "rotating universe" (2.26) which is not asymptotically flat (cf. (2.28)). This could imply a potential ambiguity in the definition of $g$. The stationary frame used in (2.19) leads to conserved string energy and hence the corresponding formula (3.44) for the $g$-factor derived in our conformally invariant model seems applicable to string states. One of the consequences of this result is that a correspondence between fundamental string states and black holes would become problematic. Indeed, the expression for the thermal average $\left\langle S_{R}\right\rangle$ calculated in ref. [10] was $\frac{1}{2} c\langle S\rangle$, where $c(1.27<c<2)$ depends on the scaling of $S$ with $M$. The formula (3.44) has an additional contribution $O(M / Q)$. Therefore, $g$ will diverge unless $M$ scales at most as $Q$. Since for black holes the charge $Q$ scales like the ADM mass $M_{\mathrm{ADM}}, M$ can scale at most as $M_{\mathrm{ADM}}$, implying a conflict in the counting of states (the correspondence between level densities requires $M \sim M_{\mathrm{ADM}}^{2}$ [50] 31

- There are at present only very few physically interesting string models on nontrivial backgrounds for which the scattering amplitudes are explicitly calculable. The present model should be one of such examples. Indeed, the tachyon vertex operator (see eqs. (2.36), (2.42) and (2.44)) is expressed in terms of exponents of $u, v$ and gaussian exponents and Hermite polynomials of the "transverse" coordinates $x^{i}$. Thus the integrals over $u, v$ can be easily carried out (as was done in the partition function in Section 4) and then the integrals over $x^{i}$ can be computed as well using the generating functional for $H_{l}$.

- We have seen that the partition function of the model (2.13) is exactly computable as a function of an arbitrary magnetic field, i.e. of free parameters $F_{i j}=$ const. In contrast to the open string theory where the tree-level (disc) partition function determines the effective (Born-Infeld) action, the tree-level (sphere) partition function $Z_{0}$ in the closed string theory is trivial when evaluated at a conformal point (it vanishes once divided over the infinite Möbius volume). That means that we cannot use it to get any information

31 This issue was recently resolved in [57]. 
about the tree-level effective action.32 At the same time, the torus partition function computed as a function of arbitrary $F_{i j}=$ const does give us the value of the one-loop effective action evaluated on our specific background (with the square root of the curvature, the antisymmetric tensor field strength and the gauge field strength all being proportional to $F_{i j}$ ), i.e., represents in certain sense a closed string analogue of the Heisenberg-EulerSchwinger action. It may be possible to use this expression to obtain some non-trivial information about the dependence of the one-loop (heterotic) string effective action on the gauge field strength.33

- The model (2.5) we have discussed in this paper is just a simple representative in a more general class of "chiral null models" corresponding to exact string solutions [53,12,. Other models in this class describe plane-fronted waves and "fundamental string" type backgrounds [54] (related, in particular, to extreme electric black holes). It may be interesting to study if some of our results can be extended to these models as well.

- We have seen that the model (2.13) is closely related to a WZW model based on a non-semisimple group. Though in this paper we did not utilise this connection to quantize and solve the theory, a more systematic current algebra approach may be useful to construct marginal operators and discover hidden symmetries.

An extension of the present work to a more general class of magnetic flux-tube backgrounds is discussed in [57].

\section{Acknowledgements}

We would like to thank G. Gibbons, E. Kiritsis, C. Kounnas, R. Metsaev, P. Olesen and K. Sfetsos for useful discussions and comments. A.A.T. is grateful to Theory Division, CERN for a hospitality during a visit when this work was at an initial stage and also acknowledges the support of PPARC and NATO Grant CRG 940870.

32 The closed string effective action $S$ always vanishes when evaluated at a conformal point. This can be considered as being a consequence of the dilaton equation of motion or a consequence of the representation $S=\left[\partial Z_{0}(t) / \partial t\right]_{t=1}$ where $t$ is the logarithm of a 2 d cutoff, $t=\log \epsilon$ [51]: since $Z_{0}$ computed at a conformal point is $\epsilon$-independent, $S$ vanishes there.

33 For example, expanding $Z$ in powers of $F_{i j}$ one may compare with the corresponding lowenergy field theory (e.g. determining a renormalisation of gauge coupling as in the open string case [52], see also [41]). 


\section{References}

[1] E.S. Fradkin and A.A. Tseytlin , Phys. Lett. B163 (1985) 123.

[2] A.A. Tseytlin, Phys. Lett. B202 (1988) 81.

[3] A. Abouelsaood, C. Callan, C. Nappi and S. Yost, Nucl. Phys. B280 (1987) 599.

[4] C.P. Burgess, Nucl. Phys. B294 (1987) 427; V.V. Nesterenko, Int. J. Mod. Phys. A4 (1989) 2627.

[5] C. Bachas and M. Porrati, Phys. Lett. B296 (1992) 77.

[6] S. Ferrara and M. Porrati, Mod. Phys. Lett. A8 (1993) 2497.

[7] E.J. Ferrer, E.S. Fradkin and V.de la Incera, Phys. Lett. B248 (1990) 281.

[8] M.B. Green, J.H. Schwarz and E. Witten, Superstring Theory (Cambridge U.P., 1987).

[9] D.J. Gross, J.A. Harvey, E. Martinec and R. Rohm, Nucl. Phys. B256 (1985) 253; Nucl. Phys. B267 (1985) 75.

[10] J.G. Russo and L. Susskind, Nucl. Phys. B437 (1995) 611.

[11] J.G. Russo, Phys. Lett. B335 (1994) 168.

[12] G.T. Horowitz and A.A. Tseytlin, Phys. Rev. D51 (1995) 2896.

[13] D. Amati and C. Klimčík, Phys. Lett. B219 (1989) 443; G. Horowitz and A. Steif, Phys. Rev. Lett. 64 (1990) 260; Phys. Rev. D42 (1990) 1950; G. Horowitz, in: Strings '90, eds. R Arnowitt et. al. (World Scientific, Singapore, 1991); H. de Vega and N. Sánchez, Phys. Rev. D45 (1992) 2783; Class. Quant. Grav. 10 (1993) 2007.

[14] A.A. Tseytlin, Nucl. Phys. B390 (1993) 153.

[15] C. Duval, Z. Horvath and P.A. Horvathy, Phys. Lett. B313 (1993) 10.

[16] E. Bergshoeff, R. Kallosh and T. Ortín, Phys. Rev. D47 (1993) 5444; E. Bergshoeff, I. Entrop and R. Kallosh, Phys. Rev. D49 (1994) 6663.

[17] C. Nappi and E. Witten, Phys. Rev. Lett. 71 (1993) 3751.

[18] E. Kiritsis and C. Kounnas, Phys. Lett. B320 (1994) 361.

[19] D. Olive, E. Rabinovici and A. Schwimmer, Phys. Lett. B321 (1994) 361.

[20] K. Sfetsos, Phys. Lett. B324 (1994) 335; Phys. Rev. D50 (1994) 2784.

[21] I. Antoniadis and N. Obers, Nucl. Phys. B423 (1994) 639

[22] K. Sfetsos and A.A. Tseytlin, Nucl. Phys. B427 (1994) 325.

[23] A.A. Kehagias and P.A.A. Meesen, Phys. Lett. B331 (1994) 77; J.M. Figueroa-O'Farril and S. Stanciu, Phys. Lett. B327 (1994) 40; A. Kehagias, "All WZW models in $D \leq 5 "$, hep-th/9406136.

[24] L. Brink, M. Green and J. Schwarz, Nucl. Phys. B198 (1982) 474; K. Kikkawa and M. Yamasaki, Phys. Lett. B149 (1984) 357; N. Sakai and I. Senda, Progr. Theor. Phys. 75 (1984) 692.

[25] E. Kiritsis, C. Kounnas and D. Lüst, Phys. Lett. B331 (1994) 321. 
[26] G.W. Gibbons and K. Maeda, Nucl. Phys. B298 (1988) 741; G.W. Gibbons, in: Fields and Geometry, Proceedings of the 22-nd Karpacz Winter School of Theoretical Physics, ed. A. Jadczyk (World Scientific, Singapore, 1986).

[27] T.H. Buscher, Phys. Lett. B194 (1987) 59; Phys. Lett. B201 (1988) 466.

[28] M. Roček and E. Verlinde, Nucl. Phys. B373 (1992) 630.

[29] A.A. Tseytlin, Nucl. Phys. B350 (1991) 395.

[30] A. Giveon, E. Rabinovici and G. Veneziano, Nucl. Phys. B332 (1989)167; K. Meissner and G. Veneziano, Phys. Lett. B267 (1991) 33; E. Álvarez, L. Álvarez-Gaumé and Y. Lozano, Phys. Lett. B336 (1994) 183.

[31] C. Klimčík and A.A. Tseytlin, Phys. Lett. B323 (1994) 305.

[32] J. Garriga and E. Verdaguer, Phys. Rev. D43 (1991) 391.

[33] L.D. Landau and E.M. Lifshitz, Quantum Mechanics (Pergamon Press, N.Y., 1977).

[34] A. Dabholkar, "Strings on a cone and black hole entropy", HUTP-94-A019, hepth/9408098; "Quantum corrections to black hole entropy in string theory", hepth/9409158; D.A. Lowe and A. Strominger, "Strings near a Rindler or black hole horizon", hep-th/9410215.

[35] E. Del Giudice, P. Di Vecchia and S. Fubini, Ann. Phys. 70 (1972) 378; K. A. Friedman and C. Rosenzweig, Nuovo Cimento 10A (1972) 53; S. Matsuda and T. Saido, Phys. Lett. B43 (1973) 123; M. Ademollo et al, Nuovo Cimento A21 (1974) 77; S. Ferrara, M. Porrati and V.L. Teledgi, Phys. Rev. D46 (1992) 3529.

[36] A. Salam and J. Strathdee, Nucl. Phys. B90 (1975) 203.

[37] N.K. Nielsen and P. Olesen, Nucl. Phys. B144 (1978) 376; J. Ambjorn and P. Olesen, Nucl. Phys. B315 (1989) 606; Nucl. Phys. B 330 (1990) 193.

[38] J. Polchinski, Commun. Math. Phys. 104 (1986) 37.

[39] W. Lerche, A. Schellekens and N. Warner, Phys. Repts. 177 (1989) 1; P. Ginsparg, in: Fields, Strings and Critical Phenomena, ed. by E. Brezin and J. Zinn-Justin (Elsevier Science Publ., 1989).

[40] W. Nahm, Nucl. Phys. B124 (1977) 121.

[41] E. Kiritsis and C. Kounnas, "Curved four-dimensional spacetime as infrared regulator in superstring theories", hep-th/9410212.

[42] A. Sen, Phys. Rev. D32 (1985) 2102; Phys. Rev. Lett. 55 (1985) 1846.

[43] C. M. Hull and E. Witten, Phys. Lett. B160 (1985) 398.

[44] S. Giddings, J. Polchinski and A. Strominger, Phys. Rev. D48 (1993) 5784.

[45] C. Johnson, Phys. Rev. D50 (1994) 4032.

[46] S. Gates, C. Hull and M. Roček, Nucl. Phys. B248 (1984) 15.

[47] C. Burgess and F. Quevedo, Nucl. Phys. B 421 (1994) 373.

[48] C. Hull and P. Townsend, Phys. Lett. B178 (1986) 187.

[49] J.J. Attick and E. Witten, Nucl. Phys. B310 (1988) 291. 
[50] L. Susskind, "Some speculations about black hole entropy in string theory", RU-93-44 (1993), hep-th/9309135.

[51] A.A. Tseytlin, Phys. Lett. B208 (1988) 221.

[52] R.R. Metsaev and A.A. Tseytlin, Nucl. Phys. B298 (1988) 109.

[53] G.T. Horowitz and A.A. Tseytlin. Phys. Rev. D50 (1994) 5204.

[54] A. Dabholkar, G. Gibbons, J. Harvey and F. Ruiz Ruiz, Nucl. Phys. B340 (1990) 33; D. Garfinkle, Phys. Rev. D46 (1992) 4286; A. Sen, Nucl. Phys. B388 (1992) 457; D. Waldram, Phys. Rev. D47 (1993) 2528.

[55] A.A. Tseytlin, Phys. Lett. B346 (1995) 55.

[56] A.A. Tseytlin, Phys. Lett. B317 (1993) 563; K. Sfetsos and A.A. Tseytlin, Phys. Rev. D49 (1994) 2933.

[57] J.G. Russo and A.A. Tseytlin, " Exactly solvable string models of curved space-time backgrounds", CERN-TH/95-20, Imperial/TP/94-95/17, hep-th/9502038. 